\title{
ABHANDLUNGEN
}

\section{Transformation der Rolle des Staates und der Grundeigentümer in städtischen Raumentwicklungsprozessen im Lichte der nachhaltigen Entwicklung ${ }^{1}$}

\author{
Peter Knoepfel / Patrick Csikos / Jean-David Gerber / Stéphane Nahrath
}

\section{Transformation of the Role of the State and of Corporate Land Owners in Urban Develop- ment Processes in Light of Sustainable Development}

Abstract: Local public land use departments encounter more and more difficulties in implementing their land use schemes due to powerful capitalistic interests of investors, land owners and even other public agents aiming at maximizing their capital investment revenues based on mortgages. The contribution calls for a comeback of a critical political debate on private land property. This debate has been practically given up in the late sixties of the last century. On the bases of large empirical research results on huge urban development projects which frequently were governed by models of a public-private-partnerships the contribution shows the role of local government as simple paver of the way for actors interested in mobilizing real estate values, it analyses new interlocked ownership structures and securitization strategies responsible for increasing lacks of transparency and responsibility and hampering political control of urban development. The article demonstrates resulting lacks of sustainability which stem from corporate private land owners' decisions on concrete land uses which are primarily driven by rapid profit interests.

Keywords: Real estate ownership, Land use planning, Sustainable Development, Capitalism, Public-Private-Partnership

Schlagwörter: Grundeigentum, Raumplanung, nachhaltige Entwicklung, Kapitalverwertung, Public-Private-Partnership

1 Dieser Beitrag basiert auf einem vom schweizerischen Nationalfonds geförderten empirischen Forschungsprojekt, das die Autoren unter dem Titel „Grundeigentum und nachhaltige Raumentwicklung. Die politischen und eigentumsbasierten Strategien der großen kollektiven Grundeigentümer der Schweiz und ihre Auswirkungen auf die nachhaltige Bodennutzung " in den Jahren 2005 2008 am Institut de hautes études en administration publique (IDHEAP) durchführten (Schweizerischer Nationalfonds für wissenschaftliche Forschung, Abteilung I (Grundlagenforschung, Projekt Nr. 100012-107833 - Gesuchsteller: Peter Knoepfel, Stéphane Nahrath; Mitbearbeiter: JeanDavid Gerber, Patrick Csikos; Mitfinanzierung: Pro Natura, Bundesamt für Statistik, Bundesamt für Raumentwicklung und Armasuisse des eidgenössischen Departements für Verteidigung, Bevölkerungsschutz und Sport - VBS). Eine zusammenfassende Übersicht der Ergebnisse findet sich bei Knoepfel et al. 2009 und bei Nahrath et al. 2009. 


\section{Einleitung}

Dieser Beitrag will aufzeigen, dass es dem raumplanenden Kommunalstaat in der Schweiz, infolge vieler Ähnlichkeiten vermutlich aber auch in Deutschland, heute kaum gelingen kann, die vielerorts postulierte Umkehr in Richtung einer nachhaltigen Raumentwicklung konkret zu vollziehen. Dies liegt nach unseren empirischen Befunden an einer seit den Anfängen der Raumordnungspolitiken bestehenden asymmetrischen Machtverteilung zwischen staatlichen Steuerungsinstanzen und immer mächtiger gewordenen Eigentümern und Investoren aller Art, an einer Umorientierung der Raumentwicklungspolitik in Richtung einer Fiskal- und Wirtschaftspolitik und an einer veränderten Struktur der Eigentümerschaft, die immer schwieriger zu steuern ist.

Diese Hypothesen werden im vorliegenden Beitrag anhand von fünf Fragen erläutert, die wir hier für die schweizerischen Verhältnisse anhand empirischen Materialien beantworten. Da die bodenrechtlichen und die raumpolitischen Bedingungen in Deutschland in vielfacher Hinsicht vergleichbar sind (Hypothekarrecht, verfassungsrechtliche Garantie des Grundeigentums, raumordnungsrechtliche Rahmenbedingungen, Verwertungszwänge für kommunales Grundeigentum), dürften die vorgetragenen konzeptionellen Grundlagen für die deutsche Diskussion durchaus anschlussfähig sein, die unseres Wissens jedoch heute (noch) weniger hohe Wellen schlägt, als in der Schweiz ${ }^{2}$. Dies gilt wohl insbesondere auch für die einleitenden Ausführungen zum Begriff der nachhaltigen Raumentwicklung, anhand dessen wir die gegenwärtigen Entwicklungstendenzen bewerten.

\section{Thematik und Fragestellung}

Wie anderswo unter Verweis auf einschlägige Autoren (Flückiger 2006; Winter 2007) gezeigt (u. a. Knoepfel 2007), beinhaltet das verfassungsmäßige Gebot der nachhaltigen Entwicklung (Schweizerische Bundesverfassung, Art. 74) eindeutig ein Primat des Erhalts der Erneuerungsfähigkeit der natürlichen Ressourcen gegenüber ökonomischen und sozialen Belangen der Nachhaltigkeit. Dementsprechend legen wir diesem Beitrag den Begriff der sog. „starken“ Nachhaltigkeit zu Grunde, die bestandsrelevante Übernutzungen natürlicher Ressourcen auch dann ausschließt, wenn der daraus ggf. resultierende ökonomische und/oder gesellschaftliche Nutzen gemeinhin als erheblich angesehen wird. Der verwendete Ansatz liegt auf der Linie einer ressourcenorientierten Nachhaltigkeitskonzeption, die „Abgleichungen“ von Einbußen auf der Ebene der natürlichen Ressourcen

2 Hier stehen heute insgesamt drei bodenrechtliche Vorlagen an: Die - umstrittene - Revision des Rauplanungsgesetzes (insbesondere: Mehrwertabschöpfung und Rückzonungspflicht für Gemeinden mit zu großen Bauzonen); die am 11. März von Volk und Ständen knapp (50,4 \%) angenommene Volksinitiative zur Beschränkung von Zweitwohnungen auf $20 \%$ und die sog. Landschaftsinitiative, die ein Moratorium für Bauzonenerweiterungen von 20 Jahren fordert und über die vermutlich im Spätsommer 2012 abgestimmt wird.

Obwohl dieser Beitrag nicht auf einer systematischen Recherche der heutigen bundesdeutschen Literatur basieren - eine solche hätte den Umfang des erwähnten Forschungsprojektes weit überschritten -, werden an verschiedenen Stellen Verweise auf Deutschland vorgenommen. Allerdings sind uns entsprechende empirische politikwissenschaftliche Studien aus Deutschland nicht bekannt. 
zugunsten ökonomischer Wertschöpfung oder sozialer Postulate ablehnt (Winter 2007).

Neuere Ansätze einer solchen ressourcenorientierten Nachhaltigkeitsforschung legen nahe, die Frage nach der nachhaltigen Raumentwicklung im Lichte der sog. „institutionellen Regime“3 natürlicher Ressourcen zu diskutieren (Nahrath 2003, 2005, 2008). Solche Regime bestehen aus der Gesamtheit privat- und öffentlichrechtlicher Regelungen, welche Nutzungsrechte an Gütern und/oder Dienstleistungen der Ressource Boden begründen. Sollen solche Regime eine nachhaltige Entwicklung im Sinne des Erhalts der Erneuerungsfähigkeit der betroffenen Ressourcen bewirken, müssen sie nach diesem Ansatz zunächst sämtliche faktisch entnommenen (gleichartigen und ungleichartigen) Güter und Dienstleistungen regeln. Dies erfolgt mittels öffentlicher Politiken und darauf abgestimmter Eigentums- und Nutzungsrechten. Sie müssen diese Entnahmen außerdem nach Maßgabe der Selbsterneuerungsfähigkeit des Ressourcenstocks beschränken. Damit sollen solche Regime übermäßige und/oder, wilde ' Nutzungen verhindern, die zu Übernutzungen führen können (Erfordernis eines hohen relativen Ausmaßes des Regimes). Schließlich müssen sie die (derart begrenzten und individuell durch entsprechende Rechtsakte zugeteilten) Nutzungsrechte untereinander mit einem geeigneten Mechanismus rechtsverbindlich koordinieren. Damit soll verhindert werden, dass die Gesamtentnahme die Reproduktionskapazität auch in Zeiten geringerer Produktion überschreitet (Erfordernis einer hohen Kohärenz).

Nachhaltigkeitsfreundlich sind nach diesem auf der institutionellen Ökonomie und der Politikanalyse basierenden Konzept (Gerber et. al. 2009) nur jene Regime, die ein hohes relatives Ausmaß und eine hohe Kohärenz aufweisen (,integrierte Regime"). Dies setzt insbesondere eine hohe (substantielle und institutionelle) Koordination der regimekonstituierenden öffentlichen Politiken („öffentliches Recht") untereinander und eine Koordination zwischen diesen Politiken und der jeweiligen eigentumsrechtlichen Grundordnung ( „Privatrecht“) voraus. Nach unseren Befunden (Knoepfel et al. 2001, 2003) ist diese Voraussetzung heute nur selten erfüllt. In vielen Fällen finden wir empirisch Regime, die zwar ein hohes „Ausmaß“4 aufweisen (rasante Zunahme öffentlicher Politiken), die indessen nur über eine mittlere oder gar eine niedrige „Kohärenz“ verfügen; in diesen Fällen sprechen wir von „komplexen Regimen“, die eher einer nicht-nachhaltigen Ressourcennutzung Vorschub leisten.

Nach diesem Ansatz werden Nutzungsrechte an Dienstleistungen und/oder Gütern, die eine Ressource zur Verfügung stellt, durch öffentliche Politiken, aber auch durch zivilrechtlich geregelte Eigentumsrechte begründet (Knoepfel 2001, S. 24 ff.; Gerber et al. 2009). Diese Ressourcen können sich wie im Falle des Bodens in privater oder in öffentlicher Hand befinden; sie können in der Schweiz aber auch dem Kantonalstaat gehören (z. B. Ressource Wasser), oder rechtlich gar keinem Eigentümer zuordenbar sein (Luft: Savary 2008, S. 49 ff.; Landschaft: Gerber 2006, S. 49 ff., Rodewald u. Knoepfel 2005, S. 30 ff.). Öffentliche Politi-

3 Im Folgenden kurz: IR-Ansatz.

4 Anzahl derjenigen real genutzten Güter und Dienstleistungen, die tatsächlich durch konkrete Regeln reguliert werden (niedriges Ausmaß = viele nicht regulierte Nutzungen). 
ken, die solche Nutzungsrechte näher umschreiben, beschränken etc., können nach diesem Konzept nur dann erfolgreich sein, wenn sie nicht auf den geballten Widerstand derjenigen gesellschaftlichen Gruppen stoßen, die daran zivilrechtlich begründete Eigentumstitel, Verfügungs- oder Nutzungsrecht innehaben (Knoepfel 1977, S. 182 ff.). Schreibt die eigentumsrechtliche Grundordnung im Zivil- und/ oder Verfassungsrecht mehr oder weniger resistente Eigentumsrechte fest, sind diese gleichermaßen wie die öffentlichen Politiken als Bestandteile institutioneller Ressourcenregime zu betrachten, die Art und Ausmaß der Nutzungsrechte und damit auch Möglichkeiten einer Über- oder Unternutzung der betroffenen Ressourcen steuern. Auch die Festschreibung solcher Eigentumsrechte ist staatliches Handeln. Dieses hat allerdings übergreifendere und längerfristigere Auswirkungen als sich oft rasch wandelnde öffentliche Politiken (Knoepfel et al. 2003, S. 51 ff.).

Vor diesem Hintergrund diskutieren wir in diesem Beitrag folgende fünf Fragen, die u. E. heute für die theoretische und praktische Debatte zur nachhaltigen Raumentwicklung von Bedeutung sind.

(1) Inwiefern hat der „historische Kompromiss“ der späten 60er-Jahre, der in der Schweiz gleichzeitig zum Aufbau umfassender Raumplanungspolitiken und zur Konsolidierung der verfassungsmäßigen Garantie des privaten Grundeigentums geführt hat, die Autonomie der Kommunen erhöht, die ihnen erlauben sollte, privates Profitstreben einzudämmen und auf diese Weise einen haushälterischen Umgang mit der Ressource Boden sicherzustellen?

(2) Inwieweit kann der planende Kommunalstaat nachhaltige Zielsetzungen nötigenfalls auch gegen den Willen der kapitalverwertungsinteressierten Eigentümer und Investoren im Rahmen von städtebaulichen Großprojekten mit oder ohne expliziten Public-Private-Partnership Modellen durchsetzen?

(3) Inwieweit trägt der planende Kommunalstaat selbst dazu bei, dass Nutzungsplanung zu einer Politik der Absicherung günstiger Rahmenbedingungen für kapitalverwertungsinteressierte Grundeigentümer, Investoren und Promotoren wird? Welche Prozesse lassen sich in dieser Umorientierung der Raumplanungs- in Richtung einer Fiskal- und Wirtschaftspolitik empirisch beobachten?

(4) Welche Akteure treten als Eigentümer und damit als primäre Ansprechpartner derart umgewandelter kommunaler Raumplanungspolitiken auf?

(5) Wie lassen sich diese neuen Eigentümer steuern (Governancestrukturen)?

\section{Der historische Kompromiss von 1969 und die Autonomie des planenden Kommunalstaates}

Während in den späten 60er- und in den frühen 70er-Jahren des letzten Jahrhunderts auf politischer Ebene (Schweizerischer Bundesrat 1967; Conradi et al. 1972; Janssen 1973; Kallenberger 1975; Rohr 1966, 1988), in den ausgehenden 70erJahre aber auch auf wissenschaftlicher Ebene (Linder 1973; Linder et al. 1979; Knoepfel 1977; Atteslander u. Hamm 1974; Binswanger 1978; Kuttler 1973; Frey 1990) in der Schweiz, in Deutschland und in anderen europäischen Ländern heftig über Ausmaß und Begrenzung des privaten Grundeigentums debattiert wurde, herrscht diesbezüglich in der Schweiz und in Deutschland seit dem einset- 
zenden Vollzug der großen Gesetzgebungswerke zur Raumplanung bzw. Raumordnung politisch bleierne Stille. Das Grundeigentum wird kaum mehr grundsätzlich hinterfragt; man meint es mittels angeblich autonomer staatlicher Planung salonfähig gemacht zu haben, womit die früher beklagten Auswüchse (Zersiedelung, Bodenspekulation, Wohnungsnot; vgl. Walter 1985; Ratz 1973; Häberli et al. 1991; Schweizerischer Bundesrat. 1967) dank politisch-administrativer Steuerungs- und Kontrollmechanismen beseitigt seien.

Gegen Mitte des ersten Jahrzehnts des 21. Jahrhunderts werden diesbezüglich in der Schweiz (Clivaz u. Nahrath 2010; Knoepfel et al. 2009; VLP-ASPAN 2008a; ARE 2010a; van Griethuysen 2010), aber auch in Deutschland (Tomerius u. Frick 2009; Einig 2005) wieder Zweifel angemeldet. Diesmal gilt die Sorge weniger dem damals dem Grundeigentum angelasteten Wohnungsproblem bzw. der als soziale Ungerechtigkeit empfundenen privaten Aneignung planungsinduzierter Mehrwerte, als vielmehr der allgemeinen Schwäche öffentlicher Raumplanung zur Durchsetzung einer nachhaltigen Raumentwicklung (ARE 2010a). Im Mittelpunkt der Kritik stehen wiederum die bereits in den 60er-Jahren als Bedrohung bezeichnete Landschaftszerstörung (Häberli et al. 1991, S. 19 ff.; Gerber 2006, S. 49 ff., 197 ff.; Rodewald u. Knoepfel 2005, S. 30 ff., Knoepfel u. Nahrath 1999, S. 756 ff.), die ungebremste Bebauung des Stadtumlandes („urban sprawling“, „étalement urbain“; vgl. Da Cunha 2005; Both 2005) und ihr als nicht nachhaltig bezeichneter Flächenverbrauch (in der Schweiz: $1 \mathrm{~m}^{2}$ pro Sekunde; vgl. ARE 2010) sowie der einhergehende und zunehmende Verbrauch anderer wichtiger Ressourcen (Flora und Fauna; Luft, Energie und Boden als Verkehrsflächen). Hinzu kommen das ungehemmte ,Ausräumen` der ländlichen Räume und die zunehmende Unfähigkeit der Ressource Landschaft, die psychische und kulturelle Identität der von rasanten Landschaftsveränderungen betroffenen Einwohner und Einwohnerinnen zu garantieren (Stremlow et al. 1998).

Der vorliegende Aufsatz will zeigen, dass diese negativen Entwicklungstendenzen ihre institutionellen Ursachen weiterhin in angeblich beseitigten Schwächen des raumplanenden Staates gegenüber einer verfassungsmäßig stark geschützten (Schweizerische Bundesverfassung, BV Art. 26) Grundeigentümerschaft haben, zu der heute auch der (vornehmlich kommunale und kantonale) Staat und eine Vielzahl kollektiver (institutioneller) Kapitalanleger gehören. Für diese Akteure hat grundpfandgesicherte Rentenbildung Vorrang vor anderen, konkreten und für die nachhaltige Entwicklung bedeutsamen Dienstleistungen, die die Ressource Boden bereitstellt. Diese Bodenleistungen sind infolge des zivilrechtlich geschützten Akzessionsprinzips (ZGB Art. 6675) für die Raumentwicklung schon deshalb von großer Bedeutung, weil sie nicht nur bauliche Flächennutzungen, sondern auch Güter und Dienstleistungen der mit dem Boden verbundenen anderen natürlichen Ressourcen umfassen (Wasser, Flora und Fauna, Luft und Untergrund sowie Landschaft; Häberli et al. 1991; Nahrath 2003). Wer in der Schweiz, in Deutschland oder in anderen westeuropäischen Ländern Bodeneigentum besitzt, ist auch

5 Schweizerisches Zivilgesetzbuch vom 10.12.1907, SR 201. Danach ist der Grundeigentümer auch Eigentümer all jener Objekte, die fest mit dem Boden verbunden sind (Gebäude, Pflanzen, Wege etc.); dazu gehört auch der Untergrund (zur heutigen Rechtslage: Laurent 2011). 
Eigentümer wichtiger kollektiver menschgeschaffener Ressourcen (Infrastrukturanlagen aller Art oder Wohnbaubestände); auch diese (nicht selten auch öffentlichen) Eigentümer müssen gemäß dem eingangs erwähnten Verfassungsgebot der nachhaltigen Entwicklung von einer entsprechend umorientierten Raumentwicklungspolitik erfasst werden. Daten zur räumlichen Entwicklung zeigen indessen, dass sich auch diese politisch potenten Infrastruktureigentümer oft gegenüber dem planenden Staat durchzusetzen vermögen (Flughäfen, Straßen, Hochspannungsleitungen etc.).

Eine neue europaweite Bodeneigentumsdebatte ist daher überfällig. Das Neue daran ist vermutlich lediglich der neue paradigmatische Kontext (Nachhaltigkeit versus soziale Gerechtigkeit und „geordnete Raumentwicklung“). Dies soll indessen nicht den Blick darauf verstellen, dass viele der neuen Nachhaltigkeitsprobleme bereits in der Bodenrechtsdebatte der 60er-Jahre angesprochen wurden (Knoepfel 1977; Schweizerischer Bundesrat 1967).

Es spricht einiges dafür, in diese Debatte auch Erfahrungen aus der Schweiz einzubringen. Ähnlich wie Deutschland (und im Gegensatz zu Frankreich) ist die Schweiz ein Land der Mieter (Schweiz: 59,4 Prozent; Deutschland: 58 Prozent; Frankreich: 45 Prozent; 20026); die hypothekarische Belastung des schweizerischen Grundeigentums ist indessen eine der höchsten der Welt (sowohl bezüglich Gesamtwerten als auch pro Einwohner ${ }^{7}$ ), weshalb der auch in andern Staaten feststellbare Kapitalverwertungsdruck auf die Raumplanung hier besonders ausgeprägt sein dürfte. Umgekehrt dürften die in diesem Beitrag dargestellten Transformationsprozesse der (eigentumsrechtlichen) Handlungssysteme des Immobiliensektors oder die starke Präsenz von (privaten und institutionellen) Investoren im öffentlichen Planungsprozess, die sich in der Raumplanung in Gestalt neuer, sog. Public-Private-Partnership-Modelle äußern, keine schweizerische Besonderheit sein. Solche Partnerschaften lassen sich auch in Deutschland und seit einiger Zeit in Frankreich feststellen, wo sich diese Arrangements oft aus einer massiven Finanzknappheit der Kommunen erklären lassen, die in der Schweiz allerdings generell ${ }^{8}$ weniger ausgeprägt ist.

Die in Europa vielerorts Ende der 60er-Jahre virulente Bodenrechtsdebatte ${ }^{9}$ hat in der Schweiz mit der Zustimmung zu den beiden Bodenrechtsartikeln (damals Art. 22ter und 22quater ${ }^{10}$ ) 1969 im Sinne eines "historischen Kompromisses“ ihren vorläufigen Abschluss gefunden. Diese Zwillingsartikel haben der Linken die Raumplanung und der Rechten die verfassungsmäßige Festschreibung des privaten Bodeneigentums und den Anspruch auf volle Entschädigung von Eigen-

6 BFS 2011b; Catte et al. 2004, S. 154.

7 Durchschnittliche Hypothekarbelastung pro Einwohner: Schweiz (2009): 70.186 CHF (SNB 2011, S. 98; BFS 2011a), Deutschland (2009): 13.912 Euro (European Mortgage Federation 2010, S. 36) und Frankreich (2009): 11.462 Euro (European Mortgage Federation 2010, S. 35).

8 Allerdings spielt die Finanzknappheit der Stadt Neuenburg im besonders untersuchten Fall des Stadiumkomplexes „La Maladière“ ebenfalls eine Rolle.

9 Das deutsche Raumordnungsgesetz stammt aus dem Jahre 1965 (8. April), die erste große Raumordnungsbehörde Frankreichs (DATAR) wurde am 14. Februar 1963 gegründet, und die große Raumplanungsreform (Loi d'orientation foncière du 30 décembre 1967) stammt aus dem Jahre 1967.

10 Heute: Art. 26 (Eigentumsgarantie) und Art. 75 (Raumplanung) der Bundesverfassung vom 19. April 1999, RS, SR 101. 
tumsbeschränkungen beschert (Aemisegger et al. 1999; Leimbacher 1995; Moor 2002). Während das in der Volksabstimmung vom Juni 1976 abgelehnte erste gesamtschweizerische Raumplanungsgesetz noch eine obligatorische Abschöpfung planungsbedingter Mehrwerte vorsah ${ }^{11}$, figuriert diese Bestimmung im zweiten, heute immer noch gültigen Raumplanungsgesetz vom 22. Juni 197912 nur noch als Kann-Formel für das kantonale Recht (RPG Art. 5). Zwar hat das Schweizerische Bundesgericht die Entschädigungsansprüche aus „materieller“ Enteignung ${ }^{13}$ in seiner bald dreißigjährigen Praxis erheblich zurückgefahren (Moor 2002). Trotzdem drohen auch heute noch Grundeigentümer nicht selten damit, im Falle ihren Interessen widersprechender Auszonungen ihrer Grundstücke aus der bekanntlich anfänglich viel zu umfangreichen Bauzone auf dem Rechtsweg Entschädigungen einzufordern. In der alltäglichen Praxis bilden solche Drohungen erhebliche Schranken für die Planungsautonomie der Gemeinden und ihre Bestrebungen, überdimensionierte Bauzonen entsprechend dem Postulat einer nachhaltigen Raumentwicklung zurückzuzonen (VLP-ASPAN 2008b), wie dies die neueste Version der Revision des Raumplanungsgesetzes (Stand: Februar 2012) für überdimensionierte Bauzonen fordert. Solche Drohungen sind heute deshalb wohl ernster zu nehmen, als es sich, nicht wie im Falle der erstmaligen Einführung der gemeindeweiten Nutzungsplanung in den 80er Jahren, in aller Regel um eigentliche Auszonungen aus rechtskräftigen Bauzonen handelt.

Hatten anlässlich des „historischen Kompromisses“ von 1969 insbesondere „staatsgläubige“ Sozialdemokraten gehofft, der „planende Staat" würde die Durchsetzung „öffentlicher“ Interessen in jedem Falle sicherstellen, so wurde in der Praxis der auch heute noch stark gemeindelastigen Nutzungsplanung jedoch bald klar, dass ihr damaliger Sieg wohl eher ein Pyrrhussieg war. Was von linken Kritikern damals als „Autonomieverlust des Staates“ (Knoepfel 1977, S. 165, 180 ff.; Grottian 1974, S. 39) oder als bedenkliche „Restriktion staatlicher Planung im kapitalistischen System“ (Ronge u. Schmieg 1972, S. 30) bemängelt wurde, dürfte sich in der Realität vielerorts bewahrheitet haben. Die in obligatorischen Volksabstimmungen auf Gemeindeebene numerisch in der Minderheit stehenden

11 Diese wird in Art. 37 des in der Volksabstimmung vom Juni 1976 abgelehnten Bundesgesetzes über die Raumplanung vom 4.10.1974 folgendermaßen umschrieben: „Mehrwertabschöpfung. 1/ Werden durch die Nutzungspläne oder sonstige planerische Vorkehren sowie durch deren Durchführung erhebliche Mehrwerte geschaffen, so sind sie durch Abgaben oder Landabtretungen von dem nach kantonalem Recht zuständigen Gemeinwesen auf den Zeitpunkt der Realisierung in angemessener Weise abzuschöpfen. 2/Die Abschöpfung von Mehrwerten, soweit sie für die Beschaffung von Realersatz zur Sicherung der bisherigen Existenzgrundlage verwendet werden, ist bis zur Realisierung der Mehrwerte der Ersatzgrundstücke aufzuschieben, wenn diese zum mittleren Preis der in der Gegend getätigten Handänderungen erworben wurden. 3/Der Ertrag der Abschöpfung ist für Raumplanungszwecke zu verwenden, ein Teil davon für einen gesamtschweizerischen volkswirtschaftlichen Ausgleich im Sinne von Artikel 45. Der Bund erlässt die notwendigen Grundsatzbestimmungen. 4/Das kantonale Recht regelt die Einzelheiten. 5/ Der Bundesrat erlässt Richtlinien über die anwendbaren Kriterien und die Höhe der Abschöpfung.“ (Zitiert in Knoepfel 1977, S. 356). Die jüngste Revision des RPG (Nationalratsbeschluss vom 29. Februar 2012) fordert eine verbindliche Mehrwertabschöpfung von mindestens $20 \%$.

12 Bundesgesetz vom 22. Juni 1979 über die Raumplanung (Raumplanungsgesetz, RPG), SR 700.

13 Formell nicht in einem Enteignungsverfahren erfolgende öffentlich-rechtliche Eigentumsbeschränkungen, die „materiell“ infolge ihrer großen Reichweite als einer Enteignung gleichkommende Beschränkungen von Nutzungs- oder Verfügungsrechten des Eigentümers betrachtet werden. 
Grundeigentümer haben es in vielen Gemeinden verstanden, auch nach Inkrafttreten des Raumplanungsgesetzes von 1979 weiterhin jene Raumentwicklung voranzutreiben, die sie unter dem früheren Regime einer auf Kernzonen beschränkten Bauzone und weitgehender Baufreiheit im Außenbereich praktizieren konnten. Anzumerken bleibt, dass das Regime der drei „dringlichen Bundesbeschlüsse über dringliche Maßnahmen in der Raumplanung "14 (Pfisterer 1975; Jagmetti 1973; Rotach 1974), die der Bundesrat und der damalige Vorsteher des Justiz- und Polizeidepartementes (Kurt Furgler) 1972 mit eiserner Hand und gegen den Widerstand der Grundeigentümer (dank der zeitlichen Befristung) durchsetzten, diesbezüglich erheblich schärfer war, weil es in Analogie zu anderen europäischen Ländern (Frankreich, Dänemark, Niederlande) keine Entschädigungen vorsah.

Der einleitend vorgestellte neue Ansatz der institutionellen Regime kommt theoretisch und praktisch zu Befunden, die den erwähnten historischen Kompromiss ex post noch stärker in Frage stellen, als dies die erwähnte kapitalismuskritische Planungsdiskussion der 70er-Jahre nahelegte. Er zeigt, dass sich unter dem damals eingeführten und heute immer noch gültigen institutionellen Bodenregime interessierte Nutzungsakteure ihre Nutzungsinteressen langfristig dadurch sichern, dass sie entweder strategisch wichtige Grundstücke zu Eigentum erwerben (nach IRAnsatz: eigentumsrechtliche Grundordnung) und sich damit auch eine privilegierte Position im öffentlichen Planungsprozess sichern (nach IR-Ansatz: öffentliche Politik).

Dieser neue Ansatz legt auch eine zweite, damals eher selten gestellte Frage nahe, die sich auf die Art der angeeigneten Güter und/oder Dienstleistungen der Ressource Boden bezieht. Darunter finden sich nicht nur verschiedenartige und unterschiedlich intensive Flächennutzungen, sondern auch die früher in Anlehnung an Karl Marx oft als „abstrakt genutzt“ (Knoepfel 1977, S. 57) bezeichneten (indirekten, weil nicht-flächenbezogenen) Bodendienstleistungen, die u. a. darin bestehen, Geldwerte zu generieren und $\mathrm{zu}$ mehren (Hypothekarwesen: Heinsohn u. Steiger 2009; Steiger 2006; de Soto 2000, van Griethuysen 2010, Vogl 2010/11, S. 130-131). Dieser Nutzung der Ressource Boden als Generator von Knappheitsrenten („Kapitalsenke“) bedienen sich heute private, halbprivate und öffentliche Investoren und sie ist durch öffentliche Politiken nur schwach reguliert bzw. begrenzt. Weil politisch die „Gefahr“ ihrer direkten Regulierung gegenwärtig in Anbetracht der davon betroffenen astronomischen Summen eher gering ist, ist deren Erwerb heute relativ sicher, sofern gleichzeitig über Maßnahmen der Raumplanung dafür gesorgt wird, dass sie nicht in Rivalität mit der Flächennutzung tritt.

Um solche Rivalitäten zu vermeiden, muss der Grundeigentümer aktiv am politischen Prozess der kommunalen Nutzungsplanung teilnehmen. Dies kann er wiederum deshalb umso besser, als ihm das Planungsrecht privilegierte Positionen im Anhörungsverfahren einräumt und er außerdem mit dem Grundeigentum über eine empirisch nachgewiesenermaßen bedeutsame Politikressource verfügt (nämlich die Politikressource „Vermögenswerte“, vgl. Knoepfel et al. 2011, S. 103 ff.).

14 Dringlicher Bundesbeschluss über Maßnahmen der Raumplanung vom 1. März 1972, Bundesblatt BBl, Heft 10, S. 761-763, 10.03.1972. 
Diese Letztere vermag einem an der Produktion öffentlicher Politiken (hier Raumplanungspolitik) beteiligten Akteur als „Zielgruppe“ dieser Politik eine gewichtige Machtposition zu verschaffen (Knoepfel et al. 2003, S. 337-338; Linder 1973, S. 197, 205). In der Politikanalyse sprechen wir von Ressourcenportfolios, die ungleich zwischen den Zielgruppen (in der Raumplanung: Eigentümer) und den Politikbegünstigten (Einwohner der betroffenen Stadtteile) verteilt sind. Diese Portfolios verschaffen den Grundeigentümern eigentliche Vetopositionen (Uhlmann u. Braun 2011, S. 35 ff). Außerdem verfügen die Eigentümer nicht nur in der Raumplanung, sondern auch als Zielgruppen vieler anderer raumrelevanter öffentlicher Politiken über diese Ressource „Vermögenswerte“, die ihnen gegenüber andern Akteursgruppen erhebliche Machtvorteile in Politikformulierungsund insbesondere in Implementationsprozessen verschaffen.

Daher vermuteten wir, dass in diesem starken Ressourcenportfolio mit ein Grund dafür zu sehen ist, dass die Eigentümer und die hinter ihnen stehenden Investoren ihre Kapitalverwertungsinteressen mittels einer Doppelstrategie durchsetzen können. Diese besteht konkret in einer Mobilisierung der allgemeinen eigentumsrechtlichen Grundordnung (Erwerb von Eigentumstiteln) und in einer (dadurch erleichterten) Einflussnahme auf den kommunalen Nutzungsplanungsprozess.

Die Ergebnisse einer Studie zu den Strategien der großen kollektiven Grundeigentümer in der Schweiz ${ }^{15}$ bestätigen diese Hypothese weitgehend: Private bodennutzungsinteressierte Akteure aller Art (insbesondere: Anlagefonds, Immobilienstiftungen, Immobiliengesellschaften), aber auch Private und Naturschutzvereinigungen wie Pro Natura (Gerber 2008a) und sogar der nutzungsplanende kommunale Staat (Knoepfel 2006) setzen in ihren Bestreben zur Mitgestaltung öffentlicher Raumplanungsprozesse trotz der seit bald dreißig Jahren bestehenden demokratischen öffentlichen Nutzungsplanung weniger auf ihre allgemeinen politischen Mitwirkungsrechte an solchen Prozessen als auf die ihnen über die Eigentümerposition zustehenden speziellen Partizipationsmöglichkeiten. Als Devise gilt: Bürgermitwirkung ist gut, Mitwirkung als Eigentümer ist besser. Konkret äussert sich dies etwa in folgenden Extrembefunden ${ }^{16}$ :

- Die Umweltorganisation Pro Natura hat trotz der (mit ihrer Unterstützung etablierten) Raumplanung nicht aufgehört, zu Schutzzwecken Eigentumsrechte an besonders schützenswerten Parzellen zu erwerben, obwohl die betroffenen Parzellen bereits durch die Raumplanung geschützt sind oder durch künftige politisch-administrative Maßnahmen geschützt werden könnten, die Pro Natura (mit Aussicht auf Erfolg) in den kommunalen Nutzungsprozess einbringen könnte (Gerber 2008a). Ähnliche Strategien verfolgen auch Umweltorganisationen anderer Länder (wie Deutschland oder die USA; Gerber 2011).

15 Kantone und Gemeinden: Gerber 2000e; Bürgergemeinden: Gerber 2008c; Schweizerische Bundesbahnen (SBB): Gerber 2008d; Armasuisse (Schweizer Armee): Gerber 2008b; Pro Natura: Gerber 2008a; Pensionskassen (Rentenversicherungsanstalten bzw. -gesellschaften): Csikos 2009a; Immobiliengesellschaften / Anlagefonds und Immobilienstiftungen: Csikos 2008; Banken und Versicherungen: Csikos 2008a; Detailhandelsketten: Fauconnet und Csikos 2009). Die Ergebnisse dieser Studien werden zusammengefasst in Nahrath et al. 2009.

16 Übrige Befunde: Siehe oben erwähnte Entschädigungsdrohungen und weiter unten. 
- Auch der planende Kommunalstaat scheint vielfach seiner eigenen Nutzungsplanung zu misstrauen, wenn er zu Schutz- oder Nutzungszwecken Grundstücke erwirbt. Denn er könnte die beabsichtigen Nutzungsziele eigentlich auch über öffentliche Planungsmaßnahmen durchsetzen (Schwab 1996; Nahrath et al. 2009).

Diese beiden Extrembeispiele zeigen, dass sich Pro Natura und selbst der kommunale Staat zunutze machen, was privat interessierte Eigentümer mit ihren Entschädigungsdrohungen ebenfalls tun: Sie verschaffen sich eine strategisch privilegierte Position im (späteren) öffentlichen Planungsprozess. Darin kommt die erwähnte Doppelfunktion des Bodeneigentums als Befähigung zur autonomen Entscheidung über die Nutzung von Gütern und Dienstleistungen, die der Boden zur Verfügung stellt, und gleichzeitig auch als Politikressource im öffentlichen Planungsprozess deutlich zum Ausdruck.

\section{Vom nutzungsplanenden Staat zum Kommunalstaat als Promotor strategischer Großbaustellen unter dem Regime von Public-Private- Partnership-Modellen}

Die von Art. 2 des schweizerischen Raumplanungsgesetzes geforderte generelle, das ganze Gemeindegebiet umfassende Flächennutzungsplanung ist heute in der Schweiz und in Deutschland weitgehend abgeschlossen. Vielerorts wurden die ursprünglichen Flächennutzungspläne aus den frühen 80er-Jahren bereits mehrfach revidiert. In der Schweiz haben diese Revisionen in den späten 80er- und in den 90er-Jahren oft zu Erweiterungen der (heute u. a. deshalb viel zu großen) Bauzonen geführt. In jüngster Zeit lassen sich in wenigen Fällen aber auch Rückzonungen beobachten (z. B. Schaffung neuer Schutz- oder Landwirtschaftszonen in ehemaligen Bauzonen: Wenger 2002; VLP-ASPAN 2010). Im Rahmen der seit Beginn des Jahrhunderts einsetzenden Verdichtungsdebatte kam es außerdem zu angehobenen Auslastungsziffern in bestehenden Bauzonen.

Insbesondere in städtischen Agglomerationen werden indessen große raumbedeutsame Umstrukturierungen heute in aller Regel nicht (mehr) über allgemeine Nutzungspläne, sondern über Sondernutzungspläne realisiert („Quartierpläne“, „Gestaltungspläne“, „plans directeurs localisés“, „plans partiels d'affectations“" etc.). Diese sind zum neuen Instrument einer auf strategische Entwicklungsgebiete städtischer Agglomerationen ausgerichteten Raumplanung geworden (Zuppinger 1990; Moor 2002; Bourdin et al. 2006; Espaces u. Sociétés 2002; Zuppinger 2012). Solche Strategien verbinden Siedlungsverdichtungen mit neuen Infrastrukturanlagen des öffentlichen Verkehrs, mit Einkaufs- und Verwaltungszentren, Sporteinrichtungen etc., und bezwecken außerdem Umnutzungen in bestehenden Siedlungsstrukturen (z. B. in Industriebrachen, „unternutzten“ Wohngebieten, Bahnhofarealen etc.). Diese nicht mehr flächendeckende, sondern die alten Nutzungspläne überlagernde und punktuelle Raumplanung führt zu einer weiteren Aufwertung der politischen und wirtschaftlichen Bedeutung der Grundeigentümer in den betroffenen Gebieten. Diese Letzteren erfahren nämlich in den trilate- 
ralen öffentlichen Planungsprozessen, die über weite Strecken eigentlichen Verhandlungen zwischen staatlichen und privater Akteuren gleichen, eine weitere Stärkung durch Koalitionsbildungen, zu denen es zwischen den betroffenen Grundeigentümern und Drittgruppen kommt. Dazu einige empirische Ergebnisse aus unseren Untersuchungen:

- Die (kommunal- oder kantonal-) staatlichen Grundeigentümer, auf deren Parzellen das neue Projekt zu stehen kommt, treten zusammen mit interessierten Investoren auf, die für solche Projekte schon deshalb unabdingbar sind, weil eine alleinige staatliche Finanzierung die Mittel der betroffenen Stadt übersteigen würde. Deshalb sucht die Stadt oft selbst aktiv Investoren und solvente Mieter für solche Objekte. Diesen muss das Projekt indessen eine vorhersehbare Kapitalrendite sicherstellen. Wie die Fallstudie Neuenburg/Maladière (Csikos 2009c) zeigt, kann bei solchen Projekten als vermittelnder Dritter ein Projektpromotor auftreten, der als Generalunternehmer die Interessen der Grundeigentümer, der Investoren und ggf. auch der Mieter bündelt und diese gegenüber den öffentlichen Behörden vertritt. Solche Projektträgerkoalitionen können außerordentlich schlagkräftig und für das Gelingen der öffentlichen Planung und deren Realisierung entscheidend sein (Logan u. Molotch 1987; Molotch 1999; Stone 1989, 1993).

- Als Gegenkoalition treten diejenigen Grundeigentümer auf, deren Grundstücke sich außerhalb des Planungsperimeters befinden und die durch das neue Projekt ggf. Wert- und Nutzungseinbußen erleiden. Unzählige Beispiele aus jüngster Zeit ${ }^{17}$ belegen die Schlagkraft solcher oppositioneller Gruppen, die auch dadurch objektive Verstärkung erfahren können, dass sich ihnen gewollt oder ungewollt beschwerdeberechtigte Umweltorganisationen anschließen. Diese Gruppe wird heute denn auch in Quartierplanungsprozessen für Großprojekte regelmäßig einbezogen; ihr Ressourcenportfolio besteht nicht nur in Vermögenswerten, sondern auch in der Ressource „Recht“, die nicht nur den Eigentümern von Nachbargrundstücken, sondern auch den Umweltorganisationen als gesetzlich anerkannten beschwerdeberechtigten Organisationen ${ }^{18}$ zusteht, und die diese Politikressource jederzeit in Gestalt koordinierter oder nicht koordinierter Beschwerden mobilisieren können.

Gegenstand dieser Aushandlungsprozesse sind etwa die durch Ausnutzungsziffern bestimmten Renditeaussichten der Investoren, die Bereitschaft ablehnender Grundeigentümer, ihre Parzellen in das neue Vorhaben einzubringen, die Beteiligung der (künftigen) Eigentums- und Nutzungsberechtigen an öffentlichen Infrastrukturaufwendungen, die Verteilung der Nutzungsrechte an den verschiedenen Komponenten der künftigen Baubestände und die künftige Governance (Entschei-

17 Paradebeispiel ist das Stadium Hardturm der Stadt Zürich (bisher nicht realisiert), in dessen Planungsverlauf die Freisinnig-Demokratische Partei des Kantons Zürich die (2005 abgelehnte) eidgenössische Volksinitiative zur Abschaffung der Verbandsbeschwerde lancierte.

18 Gemäß Verordnung vom 27 Juni 1990 über die Bezeichnung der im Bereich des Umweltschutzes sowie des Natur- und Heimatschutzes Beschwerdeberechtigten Organisationen (VBO), SR 814.076. 
dungsmechanismen für künftige Nutzungsänderungen bzw. für die (Neu-)Zuteilung von Nutzungsrechten).

Es liegt auf der Hand, dass die für Bau- und Planungsfragen zuständigen Behörden in solchen Prozessen insbesondere in kleineren und mittleren Gemeinden oft trotz einer Hilfestellung der Kantonsverwaltungen überfordert sind und die politische Entscheidungszuständigkeit an die interessierte Koalition der Grundeigentümer und Investoren abtreten („privater Gestaltungsplan“19) oder unter dem geballten Druck der Koalitionen von Befürwortern und Gegnern resignieren. Erfahrungsgemä $\beta^{20}$ segnen sie im ersteren Fall die ihnen vorgelegte Pläne unter dem mehr oder weniger aufmerksamen Auge das kontrollierenden Kantons ab; im zweiten Fall tendieren sie dazu, die Ergebnisse von Aushandlungsprozessen zwischen den beiden Koalitionen, die formell unter ihrer Ägide ausgehandelt werden, direkt in ihre öffentlichen Planungswerke zu übernehmen. In solchen Fällen verkommt staatliche Nutzungsplanung zu einer staatlichen Sanktionierung privater Nutzungsinteressen. Nicht selten werden sich die beteiligten Politiker dabei sogar auf die Schultern klopfen, weil sie im Abtausch gegen Wolkenkratzer (hohe Ausnutzungsziffern) einen Kinderspielplatz oder ein Biotop ergattert haben.

In größeren Städten (wie in jenen unserer Fallstudien) werden solche Prozesse von weit professionalisierteren Bau- und Planungsverwaltungen durchgeführt. Hier herrscht indessen auch ein größerer Investitionsdruck, weil die Kapitalverwertungsbedingungen auch mittel- und langfristig als gesichert gelten, die Standorte dementsprechend von Rating-Institutionen ${ }^{21}$ empfohlen werden und den Anlegern langfristig sichere Mieterträge in Aussicht stehen. Die Verwaltungen sind gewohnt, mit harten Bandagen zu verhandeln. Sie verfügen zunehmend auch über professionelle ökonomische Kompetenzen, die sie dazu befähigen, durch horizontale und insbesondere vertikale Verdichtungen erzielte Mehrwerte zu berechnen und diese als Gegenleistungen in Gestalt von Infrastrukturabgaben, der Gemeinde zur Verfügung gestellten Nutzungsrechten (etwa für kommunale öffentliche Einrichtungen etc.) oder in Gestalt fiskalischer Zugeständnisse (obligatorisches Steuerdomizil in der Standortgemeinde) umzumünzen verstehen. Inzwischen haben diese Behörden auch gelernt, aus dem Auftreten oppositioneller Akteure Kapital zu schlagen, indem sie den Projektträgern im Rahmen der Quartierpläne ökologische, soziale und ökonomische Zugeständnisse abringen, die aus eigens dafür eingerichteten, mehr oder weniger partizipativen Nachhaltigkeitsbeurteilungen (NHB) (ARE, 2007) resultieren.

Für beide Fälle gilt indessen, dass die Autonomie staatlicher Raumplanung, die die Konstrukteure des Raumplanungsgesetzes in der zweiten Hälfte des letzten Jahrhunderts zur Durchsetzung des „öffentlichen“ Interesses an einer geordneten Besiedelung des Landes als unabdingbar erachtet hatten (Ernst 1968; Evers 1973; Wagener 1972), in solchen Quartierplanungsverfahren offensichtlich wenig gilt

19 Beispiel: Kantone Zürich, Bern oder Waadt.

20 Einer der Autoren (Peter Knoepfel) ist seit langem Mitglied der Raumplanungskommission seiner Gemeinde mit ca. 6000 Einwohnern.

21 Z. B. Beratungsunternehmen in den Bereichen Immobilien- und Baumarkt sowie Raum- und Standortentwicklung wie „Wüest \& Partner“ oder „Informations“ oder das „Ausbildungszentrum für Immobilien (IAZI)“. 
und oft geringer ist als bei der früheren „normalen“ Flächennutzungsplanung. Denn geplant wird heute weitgehend und bewusst zugunsten ökonomischer Kapitalverwertungsinteressen der Promotoren und Investoren (Molotch 1999; Stone 1993). Das gilt insbesondere für jene Unterkategorie von Quartierplänen, die nach dem Modell einer Public-Private-Partnership aufgebaut sind. Solche Großprojekte sollen interessante Kapitalanlagemöglichkeiten für private oder öffentliche Investoren eröffnen. Seitens des planenden Kommunalstaats mag dabei auch volkswirtschaftlich motiviertes Stadtmarketing eine Rolle spielen, das der betroffenen Gemeinde Vorteile im Standortwettbewerb mit anderen Städten verschaffen soll (Harvey 2001; Mauch 2001). Das Handeln der maßgeblichen Raumplanungsbehörden wird damit von einer früher kaum dagewesenen Bevorzugung ökonomischer Belange bestimmt. Entweder betrachten diese Akteure ökonomische Aspekte als den Inbegriff des öffentlichen Interesses schlechthin, oder sie akzeptieren offen eine Dominanz der Ökonomie gegenüber sozialen und ökologischen Belangen der nachhaltigen Entwicklung, die sie nichtsdestoweniger vielfach ausdrücklich in ihren „Leitbildern“ als Handlungsmaxime verankert haben (Hollands 2008).

\section{Beitrag des planenden Kommunalstaates zur Schaffung günstiger Rahmenbedingungen für kapitalverwertungsinteressierte Grundeigentümer, Investoren und Promotoren}

Im erwähnten Forschungsprojekt wurden Fallstudien durchgeführt, die hier aus Platzgründen nicht näher zusammengefasst werden sollen ${ }^{22}$. In diesen umfangreichen Untersuchungen haben sich die in der erwähnten Vorstudie zu den Strategien der neun wichtigsten Grundeigentümer der Schweiz empirisch nachgewiesenen Strategien jedenfalls dort bestätigt, wo diese Letzteren auf den studierten „Baustellen“ präsent waren. Ihre Strategien zur Durchsetzung ihrer Nutzungsinteressen umfassten tatsächlich allesamt sowohl den Erwerb und die Nutzung von Eigentumstiteln, die ihnen eine möglichst autonome Ausübung entsprechender Nutzungsrechte und damit optimierte Kapitalverwertungsbedingungen bescherten, als auch eine direkte Einflussnahme auf den öffentlichen Planungsprozess. Als Konstante zeichnet sich auch bei öffentlichen Eigentümern die Strategie der Absicherung von Nutzungsrechten durch Eigentumserwerbs- und -verkaufsstrategien $a b$, die in unterschiedlicher Weise mit politischen Interventionen auf der Ebene der öffentlichen Raumplanung kombiniert wurden.

Diese Doppelstrategie beruht nach unseren Befunden meistens auf dem Standbein des Zivilrechts und dem Spielbein des öffentlichen Rechts. Bei keiner privaten Eigentümerkategorie konnte als Standbein die öffentlichen Raumplanungspolitik beobachtet werden ${ }^{23}$; auch öffentliche Eigentümer (Gemeinden,

22 Neuenburg (Stadiumkomplex „La Maladière“): Csikos 2009b, Biel (Einkaufszentrum Bözingenfeld/Boujean): Csikos 2009c, Bern (Verwaltungszentrum Bern-Wankdorf): Gerber 2008f und Thun (Waffenplatz): Gerber 2008g.

23 Allerdings liegt dieser Beobachtung insofern ein Bias zugrunde, als wir unsere Studie auf große Grundeigentümer konzentriert haben und z. B. nicht-landbesitzende Umweltorganisationen (wie z. B. den WWF Schweiz) ausgeschlossen haben, die ihr Handeln stärker auf öffentliche Politiken abstützen. 
Bürgergemeinden, Kantone, Schweizerische Bundesbahnen, Armee: Armasuisse) setzen oft auf das Eigentum an strategisch bedeutsamen „eigenen“ Parzellen, das ihnen eine starke Position im öffentlichen Planungsprozess verschafft. Dieser Befund schlägt sich in den Fallstudien in mehr oder weniger ausgeklügelten Rollenverteilungen zwischen kommunalstaatlichen und privaten Akteuren nieder, die nicht selten als Win-Win-Situationen und mit einer entsprechend positiven Begleitnote als Public-Private-Partnership angepriesen werden, wie sie im voranstehenden Kapitel generell umschrieben werden.

Empirisch lassen sich dabei zwei Situationen unterscheiden (Knoepfel et al. 2009, S. 133-134). Im ersten Falle will der arme Kommunalstaat mittels solcher Großprojekte infrastrukturelle Leistungsangebote aufbauen oder schlicht volkswirtschaftlichen Reichtum (mit entsprechenden Steuereinnahmen) schaffen. Zu diesem Zweck verbünden sich die staatlichen Akteure mit Eigentümern strategischer Grundstücke und suchen zusammen mit diesen Letzteren einen geeigneten Generalbauunternehmer, Investoren (z. B. Pensionskassen, Anlagefonds, Immobilienstiftungen oder Versicherungsgesellschaften) und langfristig solvente künftige Mieter (z. B. die Handelsketten Migros und Coop oder Banken). In diesem Falle besteht der Beitrag der politisch-administrativen Planungsbehörde der Gemeinden in gegenüber der allgemeinen Nutzungsplanung mehr oder weniger massiv erhöhten Ausnutzungsziffern (Ressource Recht: Legalisierung privater oder öffentlicher Gestaltungspläne) und im Einsatz ihrer Politikressource „politische Mehrheit“. Der Staat setzt damit günstige Rahmenbedingungen für eine durch Kapitalverwertungsinteressen getriebene Raumentwicklung, für die er gewissermaßen zum Türöffner und gesetzgeberischen Garanten wird (Fall Einkaufszentrum Biel: Csikos 2009c). Der Ressourcentausch besteht hier im Tausch der dem Staat zustehenden Ressource „Recht“ gegen die Grundeigentümern bzw. Investoren gehörenden Ressourcen „Geld“ und „Vermögenswerte“. Der Staat bearbeitet außerdem aktiv die Ressourcen „politische Mehrheit“ und „Konsens“, indem er potentielle Projektgegner möglichst früh in den Planungsprozess „einbezieht“, um damit die Gefahr späterer Rechtsmittelverfahren zu minimieren.

$\mathrm{Zu}$ einem weit radikaleren Bruch mit dem Konzept des autonomen, im öffentlichen Interesse planenden Gemeinwesens kommt es im zweiten Falle, den wir empirisch mit den Beispielen des Neuenburger Immobilienkomplexes „La Maladière“ (Csikos, 2009c) und des Berner Verwaltungszentrums „Wankdorf“ (Gerber, 2008f) dokumentiert haben. In beiden Fällen ist die Gemeinde gleichzeitig Planungsbehörde und Eigentümerin von Parzellen, die für die Realisierung dieser Großprojekte einen strategischen Stellenwert haben. Dabei geht es der Gemeinde im Berner Fall primär um eine politisch attraktive Inwertsetzung bisher relativ ertragslos vor sich hin dümpelnder Aktiven. Damit wollte die Stadt durch eine Anhebung der Ausnützungsziffern und die Schaffung günstiger Infrastrukturanlagen auch sich selbst „beschenken“. Im Neuenburger Fall nutzt die Gemeinde ihre Eigentümerstellung dazu, selbst zum Projektpartner zu werden und sich, ihrem Fußballclub und anderen Turnvereinen eine günstige Ausgangslage als künftigem Mieter des geplanten Objektes zu verschaffen. Um „lästige“ öffentliche Vergabe- 
verfahren zu umgehen, tritt die Gemeinde ihre Parzelle an eine eigens zu diesem Zwecke gegründete (privatrechtliche) Gesellschaft ab. ${ }^{24}$

Interessant an diesem Neuenburger Fall ist der Umstand, dass die Gemeinde den Quartierplan mit einer neugegründeten Eigentümerschaft aushandelt, an der sie selbst beteiligt ist. In äußerst geschickter Weise verstand es diese kommunale Politik außerdem, sich im Laufe des Planungsprozesses abzeichnende Projektgegner durch zahlreiche vertragliche Abmachungen an das Vorhaben zu binden, die die Bauverwaltung und das Gemeindeparlament schrittweise legalisierten. Damit managten sie die zentrale Politikressource „Konsens“ in optimaler Weise; das Projekt konnte in einer Rekordzeit von vier Jahren realisiert werden. Unsere Untersuchung zum Beitrag dieses Projekts zu einer nachhaltigen Stadtentwicklung zeigt, dass diese trotz ihrer aus gesellschaftlicher Sicht vermutlich nachhaltigen Sport-, Kultur- oder Spaßpolitik gewichtige Nachhaltigkeitslücken in Bezug auf Ökologie (relativ schwache Verdichtung auf wertvollem Bauland mit zentraler Lage) und Ökonomie (hohe Mietpreise für die städtische Verwaltung, für den Fußballclub oder für allfällige Neuwohnungsbestände) aufweist.

Außerdem verliert die Stadt mit ihrer Zustimmung zu solchen Quartierplänen ihre raumplanerische Souveränität über die betroffenen Räume für längere Zeit; sie wird als Mieterin selbst abhängig von Entscheidungen „fremder“ Investoren, die gegenüber ihren Anlegern (z. B. Pensionskassen) kontinuierlich schwarze Zahlen schreiben müssen. Und sie wird sich inskünftig wie jeder andere Nutzer mit gebundenen Händen im dornenreichen Gestrüpp des Privatrechts bewegen müssen, auch wenn sie sich dazu veranlasst sähe, öffentliche Interessen gegen die Interessen der Investoren durchzusetzen.

\section{Die „neuen“ Eigentümer}

Wer sind nun aber diese neuen Partner? Wer ist der zuständige und verantwortliche Privateigentümer? Wie ist das Eigentumsverhältnis der potenten Großeigentümer an ihren Nutzungsansprüchen an bestimmen Gütern und/oder Dienstleistungen der Ressource Boden geregelt und mit wem hat es der Staat konkret in solchen Quartierplanungsprozessen zu tun?

Bevor wir im nächsten Kapitel die Frage nach der Steuerbarkeit der beschriebenen städtebaulichen Großprojekte im Rahmen von Quartierplanungsprozessen (mit oder ohne explizite Abstützung auf Public-Private-Partnership-Modelle) behandeln, seien hier einige Strukturmerkmale der tragenden Großeigentümer angeführt, die auf eben diese Steuerbarkeit einen Einfluss haben. ${ }^{25}$

Wie im Einzelnen an anderer Stelle aufgrund dieser und einiger anderer Monografien $^{26}$ dokumentiert (Nahrath et al. 2009, S. 101 ff.), lassen sich im Rahmen

24 Andere Beispiele: La Chaux-de-Fonds oder Chur, vgl. Nahrath et al. 2009.

25 Die empirischen Ergebnisse, die wir im Folgenden zusammenfassen, haben wir im Wesentlichen in den eingangs erwähnten Untersuchungen über die Großeigentümerkategorien Banken, Anlagefonds und Immobilienstiftungen und Pensionskassen gefunden.

26 Vgl. dazu auch Theurillat et al. 2007. 
vertikal hochgradig fragmentierter realer Handlungssysteme im Immobiliensektor der Schweiz insgesamt folgende sechs Akteursgruppen unterscheiden:

- Die individuellen Anleger, die u.a. als Arbeitnehmer gesetzlich oder arbeitsvertraglich zur Zahlung von Beiträgen an ihre Rentenversicherungen verpflichtet sind. Zusammen mit den Arbeitnehmerbeiträgen fließen diese Gelder an diese Versicherer (in der Schweiz: Pensionskassen, Csikos 2009a).

- Die Pensionskassen und andere institutionelle und/oder private Anleger (Hübschle 1990): Diese Akteure sind als intermediäre Gruppe zu betrachten, die die einbezahlten Gelder sammeln, verwalten und anlegen; darunter befinden sich auch andere Anleger wie Banken und Versicherungen, die ihre Gelder in Immobilien anlegen. Diese Anleger werden oft durch Rating-Agenturen ${ }^{27}$ beraten.

- Die Anlagefonds: Diese Fonds sind (rechtlich unselbständige) Bestandteile einzelner oder mehrerer großer Finanzierungsinstituten. Sie haben als intermediäre Akteure Anteilsscheine an denjenigen juristischen Personen inne, die als formelle Eigentümer der für die mehr oder weniger nachhaltigen Stadtentwicklung relevanten Großprojekte auftreten. Solche Anlagefonds können neben Beteiligungen an diesen Eigentümern auch direktes Grundeigentum besitzen (Csikos 2008).

- Die formellen Grundeigentümer: Heute lassen sich in der Schweiz drei juristische Konstruktionen gewinnorientierten kollektiven Grundeigentums unterscheiden. Es sind dies die Immobilienstiftungen, die Immobiliengesellschaften (AG) und die bereits erwähnten Immobilienfonds (Bord 2006; Csikos 2008). Das Aktien- oder Stiftungskapital dieser juristischen Personen gehört den Aktionären bzw. den Anlegern, Stiftern oder den Fondseigentümern. Das sind die erwähnten Banken, Pensionskassen oder Versicherungen; darüber hinaus kann sich darunter aber auch eine Vielzahl privater Kleinsparer befinden. Entscheidungen über Erwerb und Verkauf von Parzellen, Gebäudebeständen oder Baurechten bzw. anderer dinglicher Rechte werden durch die Geschäftsleitungen unter der Oberaufsicht der Verwaltungs- bzw. Stiftungsräte getroffen. Im Falle der Immobiliengesellschaften partizipieren daran auch Generalversammlungen (Richtlinien der Investitionspolitik). Definitionsgemäß verfügen die Anlagefonds und die Immobilienstiftungen nicht über ein solches „legislatives" Organ. Wenn diese Eigentümer Bankkredite aufnehmen, dann wirken an solchen Entscheidungen auch die zuständigen Gläubigerbanken mit. All diese Akteure beachten die Standortempfehlungen von Rating-Agenturen, gilt doch als oberstes Ziel, den Anlegern auch mittel- und langfristig möglichst hohe Renditen sicherzustellen.

- Die Immobilienverwalter: Die formellen Eigentümer lagern die Verwaltung ihrer Immobilien in aller Regel auf spezialisierte Immobilienverwaltungsfirmen aus. Diese können entweder Tochtergesellschaften der Eigentümer, der Finanzierungsinstitute der Anleger oder spezialisierte Einheiten großer Mieter

27 Z. B. Wuest \& Partner, IAZI etc.; vgl. oben Fußnote 21. 
entsprechender Objekte sein. Mitunter gehören sie auch den die Projekte realisierenden Generalbauunternehmen.

- Die endnutzenden Mieter: Ihre Nutzungsrechte sind über mehr oder wenige exklusive und langfristige Mietverträge abgesichert. Wie bereits erwähnt, sind diese Mieter (in unseren Fallstudien: Detailhandelsketten wie Migros, Coop oder Maus-Frères, Fußballvereine oder öffentliche Verwaltungen) oft die eigentlichen Träger der Großprojekte, in deren Entwicklungsphase sie kurzfristig mitunter sogar als Eigentümer auftreten. Wie erwähnt, besteht heute in den meisten Industrie- und Gewerbebranchen die Tendenz, sich von Immobilienbesitz zu trennen und sich Nutzungsrechte durch langfristige Mietverträge zu sichern (Csikos u. Fauconnet 2009).

Diese komplexen Strukturen sind Ursache und/oder Ergebnis aus dem Zusammenwirken folgender, in unserer Studie im Einzelnen aufgezeigter fünf Entwicklungstendenzen, die allesamt eher eine negative Auswirkung auf eine nachhaltige Raumentwicklung haben dürften. Es sind dies:

- Die Finanziarisierung: Hatten früher große Unternehmungen Grundstücke als eigentliche Produktionsfaktoren erworben (Errichtung eigener Büros, Wohnbauten für Angestellte, Expansionsflächen für Industriebetriebe), so besteht heute eindeutig eine Tendenz zu ihrer Umwandlung in Finanzvermögen. Die Betriebe verkaufen sie an (möglicherweise eigens dazu gegründete, hauseigene) Immobilienfirmen, an denen sie im Gegenzug - transferierbare - Aktien oder Anteilsscheine erwerben. Teilweise werden sie dazu auch durch fiskalische Vorschriften gezwungen, weil sie die oft betriebswirtschaftlich vollumfänglich abgeschriebenen Grundstücke aktivieren müssen (Knoepfel 2006; Nahrath et al. 2008).

- Die Schaffung neuer handelbarer Wertpapiere („Verbriefungen“28: Die beschriebene Tendenz zur Finanziarisierung wird technisch und juristisch erleichtert durch die neuere Gesetzgebung, die für die beschriebenen Anlagefonds, Immobilienstiftungen und Immobiliengesellschaften ausgebaute Sondergesetzgebungen bereitstellen (Csikos 2008). Diese bringen u. a. gegenüber der früheren und recht mühsamen grundbuchbasierten Grundstückübertragung erhebliche Erleichterungen, weil sie Handänderungen in Gestalt einfacher Übertragungen von (oft börsenkotierten) Wertpapieren ermöglichen. Nach diesem Regime bleibt der formelle Eigentümer auch dann Eigentümer, wenn der Inhaber der Anteilsscheine oder Aktien diese Papiere an Dritte veräußert. Ähnlich wie im herkömmlichen Hypothekarwesen, vergibt dabei der juristische Eigentümer Nutzungsrechte an einer der zwei oben erwähnten (und mitunter rivalisierende) Dienstleistungen der Ressource Boden (Nahrath 2001), die zum einen in einem konkreten Nutzungsrecht an der Dienstleistung „Baufläche“ (mit entsprechendem Überbauungs- bzw. Wohnrecht) und zum andern an der (durch das Hypothekarrecht anerkannten, indirekten) Dienstleistung des Bodens als „Kapitalsenke“ bestehen. Wie oben erwähnt,

28 Deutscher Begriff nach Vogl 2010/11, S. 164; Französisch: „titrisation“; Englisch: „securitization“. 
wird mit dieser Letzteren der Boden für die Produktion von Wertäquivalenten in Gestalt von „Geld“ genutzt, das eine Grundrente abwirft (Binswanger 1978; Hoell 1974; Häberli et al. 1991, S. 115 ff.). Allerdings bleibt im herkömmlichen Hypothekarwesen der direkte Bezug zwischen Hypothekargläubiger (Bank) und Hypothekarschuldner (Grundeigentümer) jedenfalls im Ablösungs- oder im Konkursfall erhalten, während ein solcher zwischen den Anteilscheininhabern oder Aktionären und den real nutzungsberechtigten Akteuren nicht mehr besteht. Dementsprechend wird nachhaltigkeitsorientierte Steuerung mangels als Zielgruppen identifizierbarer Akteure erschwert. Es kommt zu einer Anonymisierung, zu einem nicht durch personelle Rücksichtnahmen gebremsten Rentenmaximierungsdruck auf die Eigentümer und Mieter und zu einer Fragmentierung der die konkrete Bodennutzung bestimmenden kollektiven Handlungssysteme des Immobiliensektors.

- Machtgewinn der intermediären Akteure und der Großmieter: Unter dem Gesichtswinkel der nachhaltigen Entwicklung ist der unbestrittene Machtzuwachs der Immobilienverwaltungsfirmen und der Anlagefonds und der Endmieter von Bedeutung. Diese zwei Akteure können die juristischen Eigentümer in die Zange nehmen. Die Ersteren (Immobilienverwaltungen) werden unter dem Druck potenter Mieter bzw. einbrechender Wohn-, Geschäfts- oder Büroflächenmärkte (insbesondere im Sinne der ökologischen und der sozialen Nachhaltigkeit gebotene) Sanierungsinvestitionen und/oder Mietpreisreduktionen einfordern. Demgegenüber werden die anderen Akteure (Pensionskassen, Banken etc.) unter dem Druck der Finanzmärkte den juristischen Eigentümern Renditenmaximierungen abverlangen. Viele raumwirksamen öffentlichen Politiken bezeichnen als ihre Zielgruppen (Knoepfel et al. 2011, S. 75-76) immer noch die juristischen Eigentümer; diese stehen auch politisch im Zentrum der öffentlichen Aufmerksamkeit. Demgegenüber sind die intermediären Akteure und die Mieter weniger sicht- und greifbar. Wie dargelegt, können sie gleichwohl Nutzungsentscheidungen zur - knappen - Ressource Boden stark beeinflussen, was für die nachhaltige Raumentwicklung von erheblicher Bedeutung ist. Das gilt insbesondere für Entscheidungen über Nutzungen nicht-flächenbezogener (indirekter) Dienstleistungen von Baubeständen (z. B. Baubestände als Lieferanten von städtebaulichen Gestaltungs- und Landschaftselementen, von Kunden für Transport-, Energie- oder Abfallunternehmungen, als Investitionsobjekte oder als Mittel zur Konjunkturankurbelung; vgl. Nicol 2009, S. 29 ff.).

- Verlängerung der Entscheidungswege: Die beschriebene Fragmentierung der Handlungssysteme des Immobiliensektors hat zur Folge, dass die Entscheidungsprozesse länger, komplexer und undurchsichtiger werden. Der erwähnte Machtzuwachs der intermediären Akteure kann leicht zu Blockierungen der juristischen Eigentümer führen, weshalb diese Letzteren von öffentlichen Politiken angeordnete oder mittels Anreizen angestrebte Verhaltensweisen (z. B. Gebäudesanierungen, Anhebung des Sozialwohnungsanteils etc.) selbst dann nicht realisieren können, wenn sie es wollten. Bei öffentlichen Eigentümern drückt sich diese Veränderung der Entscheidungswege durch Umgehung der Parlament aus. 
- Verwischung der Verantwortlichkeiten: Einhergehend mit der erwähnten Verlängerung der Entscheidungswege, lässt sich in (öffentlichen und in privaten) Handlungssystemen des Immobiliensektors eine zunehmende organisierte „Verantwortlichkeitslosigkeit“ feststellen (Csikos 2008, S. 15). Sind Mietpreissteigerungen dem juristischen Eigentümer, dem Inhaber des Anlagefonds, den Begehrlichkeiten der Anleger (Pensionskassen), den Immobilienverwaltungsfirmen oder allenfalls sogar den wichtigsten Mietern (Abwehr unliebsamer Nachbarn) zuzuschreiben? Welcher Akteur ist für die Strategie der Entwicklung von Wohnbaubeständen zuständig, die einer Immobilienstiftung, einem Immobilienanlagefonds oder einer Immobilienaktiengesellschaft gehören? Bei welchen dieser Akteure müsste dementsprechend staatliches Nachhaltigkeitshandeln einsetzen (Nicol 2009, S. 9 ff.)? Die Umstrukturierung früher vertikal integrierter Handlungssysteme des Immobiliensektors nach der Philosophie eines funktionalen „Unbundling“ (Graham u. Marvin 2001; Coen u. Heritier 2005) hat in Tat und Wahrheit zu einem noch komplexeren „Rebundling“ geführt. Dies hat vermutlich dazu beigetragen, dass die mächtigen Geschäftsführer („CEO“) in den meisten Fällen von Misswirtschaft die Verantwortung „den“ Märkten zuweisen können, die bekanntlich trotz immer salopper werdender Sprachgebräuche weder Personen noch identifizierbare verantwortliche Akteure sind.

\section{Neue Steuerungsprobleme}

Wie wirken sich diese Veränderungen der Handlungssysteme im Immobiliensektor auf den städtebaulichen Quartierplanungsprozess und auf die politischadministrative Steuerbarkeit solcher Großprojekte aus? Das erwähnte Mit- und Gegeneinander von (öffentlichen und privaten) Mietern, Immobilienverwaltungsfirmen, juristischen Eigentümern, Investoren und Banken (Anlagefonds) wird mit Sicherheit Auswirkungen auf ihre Bestandsbewirtschaftung und damit auf ihre Nachhaltigkeit zeitigen (Nicol 2009, S. 151). Auf die Rolle der politisch-administrativen Akteure als Vertreter des planenden Kommunalstaates gehen wir hier nicht mehr besonders ein; ihre relative Ohnmacht anlässlich künftiger Umgestaltungsprozesse und ihre „Gefangenheit" im originären Planungsprozess wurden oben hinlänglich beschrieben.

Die empirischen Befunde aus unseren Fallstudien vermögen (jedenfalls für den Planungsprozess) die angesprochenen Transformationsprozesse (vorerst) nur teilweise zu illustrieren. Auf die Gründe dafür wird an anderer Stelle eingegangen (Nahrath et al. 2009; Knoepfel et al. 2009). Trotzdem kündigt insbesondere der (in unserem Sample exemplarischste) Fall Neuenburg (Csikos 2009b) einige der erwähnten generellen Transformationsprozesse und deren längerfristig vermutlich negativen Folgen an. Die ersten Planungsschritte dieses Immobilienkomplexes, der neben einem Einkaufs- und Verwaltungszentrum auch ein (angeblich) dringend benötigtes Fußballstadium umfasst, setzten Mitte 2002 ein. Schon viereinhalb Jahre später wurde das Zentrum eingeweiht (Ende 2006). Bezeichnend für solche raschen, „postmodernen“ (Knoepfel et al. 2009, S. 170) Planungs- und Bauprozesse ist der Umstand, dass die Stadt sich im Rahmen einer eigens zu die- 
sem Zweck gegründeten Gesellschaft (Publicanto) die Zusammenarbeit mit dem wichtigsten künftigen Mieter (Coop), einem schweizweit tätigen Baugeneralunternehmer (HRS Real Estate) und zweier Großinvestoren (Pensionskassen Publica und Swisscanto) sicherte. Sie trat dieser Gesellschaft ihr (parzellarisch bereinigtes) Grundeigentum zu einem symbolischen Preis von einem Franken ab. Wie vereinbart, finanzierten diese beiden Pensionskassen (über die Gesellschaft) den gesamten Komplex, an dem die Stadt schließlich zu 17,2 Prozent (im Grundbuch eingetragene) Miteigentümerin wird; die restlichen 82,8 Prozent verbleiben in der Hand der neuen Gesellschaft (und damit in der Hand der Pensionskassen).

Die Stadt konnte damit ihr ca. $30.000 \mathrm{~m}^{2}$ umfassendes Bauland quasi gratis in einen riesigen Baukomplex umwandeln, an dem sie heute Miteigentümerin ist. Sie hat allerdings (wie Coop) für ihre Flächennutzungen (Feuerwehrdienst, Turnhallen und Sportstadium) den Investoren jährlich beträchtliche Zinsleistungen zu erbringen. Die Genossenschaft Coop, auf die die Stadt übrigens erst nach gescheiterten Verhandlungen mit ihrer Konkurrentin (Migros) als potenten künftigen Mieter stieß, hat von Anfang an auf eine Mieter- und nicht auf eine Eigentümerstrategie gesetzt (wie sie das entsprechend ihrer neueren Immobilienstrategie auch anderswo tut). Die Genossenschaft kontrolliert im Übrigen die Auswahl der anderen im Zentrum als Untermieter zugelassenen Geschäfte. Im Grundbuch angemerkt ist schließlich ein Kaufrecht der Stadt für den Fall eines Verkaufs der Anteile an der Gesellschaft Publicanto durch eine oder beide beteiligten Pensionskassen.

Der Fall zeigt einige der oben angesprochenen Umwandlungsprozesse von Handlungsstrukturen des Immobiliensektors. Es sind dies die Umwandlung von Eigentum in Wertpapiere (förmliche juristische Eigentümerin ist die Gesellschaft Publicanto und nicht die beiden Investoren), die bedeutende Rolle intermediärer Akteure (Coop als Mieter und der Generalunternehmer HRS-Real Estate), die Verlängerung der Entscheidungswege („Umweg“ über die einfache Gesellschaft) und die Verwischung der Verantwortlichkeiten (Bedeutungsverlust des Parlaments und der kommunalen Planungsverwaltung nach Verabschiedung des Quartierplans und Übertragung von Entscheidungszuständigkeiten über die Bodennutzung im ehemals öffentlichen Raum von der Stadt an die privatrechtliche Gesellschaft).

Nach unseren Befunden stellt das im einschlägigen Quartierplan als lokalem regulatorischem Arrangement (Aubin 2007; de Buren 2011; Schweizer 2010, S. 58 ff.) konkretisierte Regime auch nach der Umwandlung der früheren Bodennutzung ein relativ integriertes (und nach dem oben erwähnten theoretischen Konzept nachhaltigkeitsfreundliches) Regime dar. Denn der Gesellschaftervertrag regelt in der Tat die Nutzungsrechte sämtlicher (anlässlich des Planungsprozesses tatsächlich interessierter) Partner in recht detaillierter Weise und er legt ebenfalls jene Mechanismen präzise fest, die im Falle einer Veränderung dieser Nutzungsrechte zum Tragen kommen sollten. Dieses Arrangement begründet eine Governancestruktur, die für die heutigen und für die künftigen Nutzungsrechte der beteiligten Akteure klare Regeln setzt. Es weist außerdem auch genügend Flexibilität auf, um künftige Veränderungen oder Nutzungsrivalitäten angemessen und unter Beteiligung sämtlicher Partner zu regulieren. Soweit ersichtlich dürfte das nicht nur für die direkte flächen- sondern auch für nicht-flächenbezogene (indirekte) Nutzungsansprüche externer Akteure gelten (wie Energielieferanten, Abfallabneh- 
mer, öffentlicher und privater Verkehr, zugelassene (Neu-)Investoren oder gar rechtlich geschützte Nutzungsansprüche an der durch den Baukörper neu entstandenen städtischen Landschaft; vgl. Nicol 2009, S. 29 ff.). Diese werden jedenfalls teilweise ebenfalls direkt in Bestimmungen des Quartierplans oder in anderen Dokumenten geregelt.

Entgegen der allgemeinen Hypothese des IR-Ansatzes führt indessen das relativ integrierte lokale Arrangement gegenüber dem früheren Zustand zu den oben erwähnten Nachhaltigkeitslücken im ökonomischen und sozialen Bereich. Dieser Umstand stellt die allgemeine Hypothese des IR-Ansatzes teilweise in Frage; er legt nahe, für die Qualifizierung nachhaltigkeitsfreundlicher Arrangements neben ihrem Ausmaß und ihrer Kohärenz eine weitere inhaltliche Dimension zu entwickeln, die sich an einer ausgewogenen Zuteilung der (neuen) Nutzungsrechte an im Planungsprozess untervertretene Nutzungsinteressen orientiert ${ }^{29}$.

Wie bereits erwähnt, bindet indessen dieses feingliedrige (und nicht vorbehaltslos als nachhaltigkeitsfreundlich zu bezeichnende) Vertragswerk die - beschenkte - Stadt und es beschränkt auch ihre Planungssouveränität für künftige Nutzungsänderungen. Dem planenden Kommunalstaat sind damit die Hände für lange Zeit gebunden. In der Gesellschaft Publicanto sind die Pensionskassen schon infolge der Anteilsverteilung ein weitaus stärkerer Partner als die Stadt. Grundlegende Nutzungsänderungen mögen heute zwar als unrealistisch erscheinen. Was aber gilt, wenn sich die Mieter (wie im Falle des Bieler Einkaufszentrums geschehen: Csikos 2009c) „gezwungen“ sähen, sich aus dem Neuenburger Geschäft zurückzuziehen, womit sie auch die Rentabilität der Kapitalanlage der Pensionskassen und damit die Sicherheit „unserer" Renten in Frage stellen würden? Inwiefern könnte die Stadt, etwa veranlasst durch lufthygienische, andere umwelt- oder nachhaltigkeitsorientierte Postulate und dem Auftreten darauf ausgerichteter neuer Nutzungsakteure ${ }^{30}$ eine Reduktion der 900 genehmigten Parkplätze durchsetzen? Könnte sich in einem solchen Falle eine entsprechend umorientierte städtische Politik tatsächlich gegen privatrechtliche Bindungen der Stadt durchsetzen? Oder was geschähe im Falle eines Konkurses des Fußballclubs Xamax ${ }^{31}$ der ebenfalls Mieteinnahmen generiert?

Vieles deutet daraufhin, dass Governancestrukturen, die nach dem Modell einer Public-Private-Partnership aufgebaut sind, schon auf Grund divergierender Interessen der Partner wenig krisensicher sind (Pollitt u. Bouckaert 2004; Hughes 2003). Denn sie laufen aus den genannten Gründen Gefahr, zu (definitionsgemäß einer nachhaltigen Entwicklung nicht förderlichen) komplexen Arrangements zu mutieren und können damit (bildlich gesprochen) zum schrittweisen „Zerfall“ der Bau- und Geschäftssubstanz der betroffenen Objekte beitragen (Nicol 2009, S. 91). Das immobilisierte Kapital ist eben nicht so „immobil“, wie es scheinen mag; die Umwandlung von Eigentum in Wertpapiere erschwert (absichtsgemäß)

29 Diese Dimension wurde in der referierten empirischen Studie nicht konzeptualisiert und bleibt daher empirisch unbeantwortet. Sie wird in einer neuen IR-Studie zu patrimonialen Ressourcen (Labelisierung von Lebensmitteln besonderer Herkunft und Landschaften) als Erweiterung des IR-Ansatzes neu verwendet (vgl. Boisseaux et al. 2012).

30 Deren Absicht bestünde genau darin, die bezeichneten realen Nachhaltigkeitslücken zu schließen.

31 Tatsächlich eingetreten im Sommer 2011; ähnliches droht 2012 auch dem Genfer Club Servette. 
eine langfristige Kapitalbindung, wie sie für den Bestandschutz der Ressource Boden notwendig wäre.

\section{Schluss: Nachhaltigkeitsdefizite infolge überhitzter, renditengetriebener Bodennutzungen}

Quartierplanungs- und Bauprozesse, die nach dem vorgestellten „Neuenburger Konzept" und damit nach dem Modell einer Public-Private-Partnership erfolgen, sind in der Schweiz erst seit Anfang dieses Jahrhunderts en vogue (u. a. Sportund Einkaufszentrum La Praille in Genf, Fußballstadium Hardturm in Zürich, Zürich Westbahnhof, etc.). Sowohl Investoren, als auch gewichtige Mieter, spezialisierte Generalbauunternehmungen und große Kommunalverwaltungen verfügen über ein beachtliches Regulierungswissen, weshalb diese Prozesse in der Regel wohl zu kurz- und mittelfristig soliden, (im bisherigen Sinne unserer IR-Definition) relativ integrierten lokalen Bodennutzungsarrangments führen dürften.

Allerdings stehen wir heute erst am Anfang einer großen Umorientierung der Raumentwicklung in Richtung Nachhaltigkeit (ARE 2011) und Einbrüche der Finanzwirtschaft bzw. Erhöhungen der heute außerordentlich tiefen Hypothekarzinssätze ${ }^{32}$ sind erfahrungsgemäß in Zukunft nicht auszuschließen. Es steht deshalb keinesfalls fest, dass die neu entstandenen Steuerungsstrukturen für solche raumplanerischen Großprojekte auch langfristig krisenfest sind und damit auch für künftige Generationen Nachhaltigkeit versprechen können. Aufgrund unserer Ergebnisse und anderer Überlegungen eher ökonomischer Provenienz zu entsprechenden Erfahrungen zur Verlässlichkeit solcher Konstruktionen in Frankreich (Athias 2009), steht wohl eher das Gegenteil zu vermuten. Solange rankinggesteuertes Investieren und finanzmarktgesteuertes Desinvestieren die Raumentwicklung derart massiv erschüttern können, wie dies die heutige Bodenrechtsordnung und deren recht gefahrenreiche Verkoppelung mit den neuen Finanzierungsinstrumenten zulässt, wird auch die beste Absicht für eine Umorientierung der Raumentwicklung in Richtung Nachhaltigkeit kaum erfolgreich sein.

Erfolgversprechender sind nach den in diesem Aufsatz dargelegten Überlegungen Strategien, die die eigentumsrechtliche Grundordnung der Ressource Boden ins Zentrum stellen, die wir vor dem Hintergrund der obigen Ausführungen als eigentliches Stiefkind des historischen Kompromisses von 1969 betrachten. Wer sich die Mühe nimmt, die wohl weitgehend vom Spezialisten des germanischen Rechts Eugen Huber verfasste Botschaft des Bundesrates zum ZGB von $1905^{33}$ oder Abhandlungen seiner Berufskollegen zu den Veränderungen des Zivilrechts im 18. Jahrhundert ${ }^{34} \mathrm{zu}$ lesen, wird leicht erkennen, dass dabei insbesondere eine sozialverträglichere Regulierung des Hypothekarwesens anzustreben ist, die diese

32 Auf diese Gefahr hat jüngst (März 2012) eine interne Arbeitsgruppe der Finanzverwaltung des Bundes in einem Bericht zu den Risiken des heutigen Finanzsystems hingewiesen; die Gruppe empfiehlt, das Hypothekarwesen und die darauf zurückzuführende Überschuldung neu zu überdenken.

33 Botschaft des Bundesrates zum Entwurf des schweizerischen Zivilgesetzbuch vom 28. Mai 1904.

34 Hedemann 1930. 
Art der Geldanlage stärker beschränkt ${ }^{35}$ und ggf. an raumplanerische Vorgaben bindet ${ }^{36}$. Wie die US-amerikanischen Erfahrungen mit der Subprime Rate Crisis nach 2007 oder das Platzen der Immobilienblase Spaniens zeigen, zeitigen in deren Gefolge langfristig leergestellte Gebäudebestände durchaus greifbare negative Folgen auf die reale Raumbewirtschaftung und damit auch auf die Raumordnungspolitik.

Im Sinne des theoretischen Konzepts der institutionellen Regime stehen im Falle (städte-) baulicher Bodennutzungen auch sozial-, umwelt- und wirtschaftsverträglichere Regulierungen der immer bedeutsameren kollektiven Dienstleistungen anderer nicht-flächenbezogener Art (Nicol 2009) der Ressource Boden zur Debatte. Denn die Zuteilung der Verfügungsrechte über diese Bodendienstleitungen ist heute eine entscheidende Komponente der politischen und gesellschaftlichen Machtverteilung unter den Akteuren raumplanerischer Prozesse in städtischen und ländlichen Räumen unserer europäischen Gesellschaften. Nachhaltigkeitsorientierte Umsteuerung der räumlichen Entwicklung muss daher paradoxerweise besonders diese nicht-flächenbezogenen Dienstleistungen der Ressource Boden stärker zum Gegenstand haben, als die Raumplanung heutiger Prägung.

\section{Literatur}

Aemisegger, Heinz, Alfred Kuttler, Pierre Moor und Alexander Ruch (Hrsg.). 1999. Kommentar zum Bundesgesetz über die Raumplanung. Zürich: Schulthess.

ARE (Bundesamt für Raumentwicklung). 2007. Nachhaltigkeitsbeurteilung von Projekten auf der Ebene der Kantone und Gemeinden, ein Leitfaden.

ARE (Bundesamt für Raumentwicklung). 2010a. Zum Stand der Raumentwicklung in der Schweiz.

ARE (Bundesamt für Raumentwicklung). 2010b. Raumentwicklungskonzept.

ARE (Bundesamt für Raumentwicklung). 2011. Nachhaltige Raumentwicklung Schweiz.

Athias, L.aure. 2009. Responsabilité politique et design contractuel des Partenariats Public Privé. Revue économique 60:1011-1021.

Atteslander, Peter, und Bernd Hamm (Hrsg.). 1974. Materialien zur Siedlungssoziologie. Köln: Neue Wissenschaftliche Bibliothek.

Aubin, David. 2007. L'eau en partage. L'activation des règles dans les rivalités d'usages en Belgique et en Suisse. Brüssel: Peter Lang.

BFS (Bundesamt für Statistik). 2011a. Spartätigkeit und Hypothekarforderungen nach Domizil und Standort der Pfandobjekte - Ende 2002-2009, tab. [T 12.4.2.3]. In Statistisches Lexikon der Schweiz (online). Neuchâtel: BFS.

35 Vgl. zu den Folgen ungehemmter Verbriefungen im Immobilienwesen eindrücklich: Vogl 2010/11, S. $164 \mathrm{ff}$.

36 Wir vermuten, dass die erwähnten Investitionsempfehlungen privater Ratinggesellschaften in der Tat bedeutsame Auswirkungen auf raumplanerische Schwerpunktsetzungen bzw. Entleerungsprozesse und auf die entsprechende Bodenpreisentwicklung haben; faktisch fühlen sich „gute“ geschäftsführende Organe von Investoren heute geradezu verpflichtet, diese Empfehlungen zu befolgen, um sich nicht der Kritik ihrer Aufsichtsgremien auszusetzen. 
BFS (Bundesamt für Statistik). 2011b. Eigentumsverhältnisse in Prozent der jeweiligen Bevölkerungsgruppe - 1996-2008, tab. [su-d-13.5-as-1.2.3]. In Statistisches Lexikon der Schweiz (online). Neuchâtel: BFS.

Binswanger, Hans Christoph. 1978. Eigentum und Eigentumspolitik. Zürich: Schulthess.

Boisseaux, Stéphane, Laurent Tippenhauer, Melaine Leassle und Peter Knoepfel. 2012. Labellisation du patrimoine: une approche néo-institutionnelle. Schweizerische Zeitschrift für Politische Wissenschaft (eingereicht).

Bord, Mélanie 2006. Analyse juridique des formes de propriété et de propriétaires collectifs en Suisse, IDHEAP Working Paper 10/2006. Lausanne: Hochschulinstitut für öffentliche Verwaltung.

Both, Jean-François. 2005. Métropolisation et étalement urbain: un nouveau régime d'urbanisation. In Enjeux du développement urbain durable: transformations urbaines, gestion des ressources et gouvernance, Hrsg. Antonio Da Cunha, Peter Knoepfel, JeanPhilippe Leresche und Stéphane Nahrath, 39-53. Lausanne: Presses Polytechniques.

Bourdin, Alain, Marie-Pierre Lefeuvre und Patrice Melé (Hrsg.). 2006. Les règles du jeu urbain: entre droit et confiance. Paris: Descartes \& Cie.

Catte, Pietro, Nathalie Girouard, Robert Price und Christophe André. 2004. La contribution des marchés du logement à la résilience économique. Revue économique de l'OCDE 2004:139-173.

Clivaz, Christophe, und Stéphane Nahrath. 2010. Le retour de la question foncière dans l'aménagement des stations touristiques alpines en Suisse / The return of the property question in the development of Alpine tourist resorts in Switzerland. Revue de géographie alpine / Journal of Alpine Research 98: Beitrag 2.

Coen, David, und Adrienne Héritier (Hrsg.). 2005. Refining Regulatory Regimes. Utilities in Europe. Oxon: Edward Elgar.

Conradi, Peter, Hartmut Dieterich und Volker Hauff. 1972. Für ein soziales Bodenrecht. Frankfurt a. M.: Europäische Verlagsanstalt.

Csikos, Patrick. 2008. Les stratégies foncières des grands propriétaires fonciers collectifs: les cas des sociétés immobilières, fonds de placement immobilier et fondations de placement, IDHEAP Working Paper 10/2008. Chavannes-près-Renens: Hochschulinstitut für öffentliche Verwaltung.

Csikos Patrick. 2009. Les stratégies foncières des grands propriétaires fonciers collectifs: les cas des compagnies d'assurances et des banques, IDHEAP Working Paper SF 9/2009. Chavannes-près-Renens: Hochschulinstitut für öffentliche Verwaltung.

Csikos, Patrick. 2009a. Les stratégies foncières des grands propriétaires fonciers collectifs: le cas des caisses de pensions, IDHEAP Working Paper SF 7/2009. Chavannes-prèsRenens: Hochschulinstitut für öffentliche Verwaltung.

Csikos, Patrick. 2009b. Les stratégies foncières des grands propriétaires fonciers: étude de cas du complexe immobilier de «La Maladière» à Neuchâtel, IDHEAP Working Paper Etudes 1/2009. Chavannes-près-Renens: Hochschulinstitut für öffentliche Verwaltung. Csikos, Patrick. 2009c. Les stratégies foncières des grands propriétaires fonciers: étude de cas du «Centre Boujean» à Bienne, IDHEAP Working Paper Etudes 3/2009. Chavannes-près-Renens: Hochschulinstitut für öffentliche Verwaltung.

Csikos, Patrick, und Marie Fauconnet. 2009. Les stratégies foncières des grands propriétaires fonciers collectifs : le cas des entreprises de commerce de detail, IDHEAP Work- 
ing Paper SF 3/2009. Chavannes-près-Renens: Hochschulinstitut für öffentliche Verwaltung.

Csikos P., Gerber J.-D. 2009. L'impact de la nouvelle gestion publique sur la politique de gestion du patrimoine foncier et immobilier de la Confédération. In Réformes de politiques institutionnelles et action publique / Reformen institutioneller Politiken und Staatshandeln, Hrsg. Peter Knoepfel, 231-254. Lausanne, Bern: Presses Polytechniques et Universitaires Romandes.

Da Cunha, Antonio. 2005. Régime d'urbanisation, écologie urbaine et développement urbain durable: vers un nouvel urbanisme. In Enjeux du développement urbain durable: transformations urbaines, gestion des ressources et gouvernance, Hrsg. Antonio Da Cunha, Peter Knoepfel, Jean-Philippe Leresche und Stéphane Nahrath, 12-38. Lausanne: Presses Polytechniques.

de Buren, Guillaume. 2011. Arrangements régulatoires, IDHEAP Working paper (im Erscheinen).

Einig, Klaus. 2005. Regulierung des Siedlungsflächenwachstums als Herausforderung des Raumordnungsrechts, DISP 160:48-57.

European Mortgage Federation. 2010. HYPOSTAT 2009 - A Review of Europe's Mortgage and Housing Markets. Brüssel: European Mortgage Federation.

Ernst, Werner. 1968. Raumordnung als Aufgabe der planenden Gesetzgebung und Verwaltung. In Planung III, Hrsg. Joseph H. Kaiser, 129-172. Baden-Baden: Nomos.

Espaces et Sociétés, 2002. Projet urbain, maîtrise d'ouvrage, commande. Espaces et sociétés (105/106).

Evers, Hans-Ulrich. 1973. Das Recht der Raumordnung. München: Goldmann.

Flückiger, Alexandre. 2006. Le développement durable en droit constitutionnel suisse. Umweltrecht in der Praxis / Droit de l'environnement dans la pratique 20:471-526.

Frey, René L. 1990. Boden: Zwischen Markt und Politik. In ders.: Städtewachstum, Städtewandel. Eine ökonomische Analyse der schweizerischen Agglomerationen. Basel / Frankfurt a. M.: Helbing \& Lichtenhahn.

Gerber Jean-David. 2011. The Difficulty of Integrating Land Trusts in Land Use Planning. Landscape Urban Planning, doi:10.1016/j.landurbplan.2011.11.002.

Gerber, Jean-David. 2006. Structures de gestion des rivalités d'usage du paysage, une analyse comparée de trois cas alpin. Zürich: Rüegger.

Gerber, Jean-David. 2008a. Les stratégies foncières des grands propriétaires fonciers collectifs: le cas de Pro Natura, IDHEAP Working Paper SF 6/2008. Chavannes-prèsRenens: Hochschulinstitut für öffentliche Verwaltung.

Gerber, Jean-David. 2008b. La propriété foncière du Département fédéral de la défense, de la protection de la population et des sports (DDPS) gérée par Armasuisse immobilier, IDHEAP Working Paper 10/2008. Chavannes-près-Renens: Hochschulinstitut für öffentliche Verwaltung.

Gerber, Jean-David. 2008c. Les stratégies foncières des grands propriétaires fonciers collectifs: le cas de des bourgeoisies de Berne, Soleure et Coire, IDHEAP Working Paper SF 2/2008. Chavannes-près-Renens: Hochschulinstitut für öffentliche Verwaltung.

Gerber, Jean-David. 2008d . Les stratégies foncières des grands propriétaires fonciers collectifs: le cas des CFF, IDHEAP Working Paper SF 4/2008. Chavannes-près-Renens: Hochschulinstitut für öffentliche Verwaltung. 
Gerber, Jean-David. 2008e. Les stratégies foncières des grands propriétaires fonciers: étude de cas du pôle de développement économique du Wankdorf, IDHEAP Working Paper Etudes 2/2008. Chavannes-près-Renens: Hochschulinstitut für öffentliche Verwaltung.

Gerber, Jean-David. 2008f. Les stratégies foncières des grands propriétaires fonciers: étude de cas de la place d'armes de Thoune, IDHEAP Working Paper Etudes 4/2008. Chavannes-près-Renens: Hochschulinstitut für öffentliche Verwaltung.

Gerber, Jean-David, Peter Knoepfel, Stéphane Nahrath, und Frédéric Varone. 2009. Institutional Resource Regimes: Towards Sustainability Through the Combination of Property-Rights Theory and Policy Analysis. Ecological Economics 68:798-809.

Graham, Steve, und Simon Marvin. 2001. Splintering Urbanism. Networked Infrastructures, Technological Mobilities and the Urban Condition. London: Routledge.

Grauhahn, Rolf R. 1973. Strukturwandlungen planender Verwaltung - Beispiel der Münchner Stadtentwicklungsplanung. In Gesellschaftspolitische Planung, Hrsg. B. Schäfers, 231-252. Stuttgart: Enke.

Grottian, Peter. 1974. Strukturprobleme staatlicher Planung. Hamburg: Hoffmann und Campe.

Häberli, Rudolf, Claude Lüscher, Brigitte Praplan Chastonay und Christian Wyss. 1991. Boden-Kultur. Vorschläge für eine haushälterische Nutzung des Bodens in der Schweiz. Schlussbericht des Nationalen Forschungsprogrammes (NPF) 22 „Nutzung des Bodens in der Schweiz“. Zürich: vdf Hochschulverlag.

Harvey, David. 2001. Spaces of Capital: Towards a Critical Geography. New York: Routledge.

Hedemann, Justus W. 1930. Die Fortschritte des Zivilrechts im XIX. Jahrhundert. 2. Teil, 1. Halbband. Berlin: Sauer \& Auvermann.

Heinsohn, Gunnar, und Otto Steiger. 2009. Eigentum, Zins und Geld: Ungelöste Rätsel der Wirtschaftswissenschaft. Marburg: Metropolis-Verlag.

Hoell, Günter. 1974. Die Grundrente und die Entwicklung des Kapitalismus in der Landwirtschaft. Berlin: Dietz.

Hollands, Robert G. 2008. Will the Real Smart City Please Stand Up? City 12: 303-320.

Hübschle, Jörg, Hans-Rudolf Schultz, August Hager und Urs Müller. 1990. Sozialversicherungssparen und Immobilienmarkt, Bericht 64 des Nationalen Forschungsprogrammes „Boden“. Stuttgart: IRB Verlag.

Hughes, Owen E. 2003. Public Management and Administration: An Introduction. New York: Palgrave Macmillan.

Jagmetti, Riccardo L. 1973. Les mesures urgentes en matière d'aménagement du territoire. In Treizième Journée Juridique, Hrsg. R. Oberson, J. Thorens und Riccardo L. Jagmetti, 51-80. Genf: Librairie de l'Université Georg \& Cie.

Janssen, Jörn. 1973. Zweihundert Jahre Bodenreform - für Liberale, Kleinbürger, Reformer, Utopisten und Interessenten. PR-Hefte Nr. 9, Bodenrecht und Bodenpolitik in der BRD. Köln.

Kallenberger, Werner. 1975. Literatur zum Eigentum, zum Bodenproblem und zur Bodenpolitik (Mskr. verv.), erstellt für die Schweizerische Gesellschaft für ein Neues Bodenrecht, Bern.

Knoepfel, Peter. 1977. Demokratisierung der Raumplanung: grundsätzliche Aspekte und Modell für die Organisation der kommunalen Nutzungsplanung unter besonderer 
Berücksichtigung der schweizerischen Verhältnisse. Schriften zum öffentlichen Recht Bd. 321. Berlin: Duncker \& Humblot.

Knoepfel, Peter. 2006. Der Staat als Eintümer. In Contribution à l'action publique / Beiträge zum öffentlichen Handeln, Hrsg. Jean-Loup Chappelet, 145-162. Lausanne: Presses polytechniques et universitaires / Bern: Haupt.

Knoepfel, Peter. 2007. La création de droits d'usages de ressources naturelles - questions aux juristes. Umweltrecht in der Praxis / Droit de l'environnement dans la pratique No 1, 2007: 115-153.

Knoepfel, Peter (Hrsg.). 2007. Environmental Policy Analyses. Learning from the Past for the Future - 25 Years of Research. Berlin: Springer.

Knoepfel, Peter, Johan Imesch, Corinne Larrue und Mathieu Bonnefond. 2011. Conceptual Framework for Studies on Local Regulatory Arrangements (LRA) for Selected (new) Activities in Rural Areas, IDHEAP Working Paper (online) 2/2011. Chavannes/ Lausanne: Hochschulinstitut für öffentliche Verwaltung.

Knoepfel, Peter, Ingrid Kissling-Naef und Frédéric Varone (Hrsg.). 2001. Institutionelle Regime für natürliche Ressourcen: Boden, Wasser und Wald im Vergleich. Basel u. a.: Helbing \& Lichtenhahn.

Knoepfel, Peter, Ingrid Kissling-Naef und Frédéric Varone (Hrsg.). 2003. Institutionelle Regime natürlicher Ressourcen in Aktion, Oekologie und Gesellschaft Nr. 19. Zürich: Rüegger.

Knoepfel, Peter, Corinne Larrue, Frédéric Varone und Sylvia Veit. 2011. Politikanalyse. Leverkusen: Barbara Budrich - UTB.

Knoepfel, Peter, und Stéphane Nahrath. 1999. Umwelt- und Raumordnungspolitik / Politique de l'environnement et de l'aménagement du territoire. In Handbuch der schweizer Politik, Hrsg. Ulrich Klöti, Peter Knoepfel, Hanspeter Kriesi, Wolf Lindner, Yannis Papadopoulos und Pascal Sciarini, 756 ff. Zürich: Verlag Neue Zürcher Zeitung.

Knoepfel, Peter, Stéphane Nahrath und Frédéric Varone. 2007. Institutional Regimes for Natural Resources: An Innovative Theoretical Framework for Sustainability. In Environmental Policy Analyses. Learning from the Past for the Future - 25 Years of Research, Hrsg. Peter Knoepfel, 455-506. Berlin: Springer.

Knoepfel, Peter, Stéphane Nahrath, Patrick Csikos und Jean-David Gerber. 2009. Les stratégies politiques et foncières des grands propriétaires fonciers en action - études de cas. Cahier de l'IDHEAP 247. Chavannes-près-Renens: Hochschulinstitut für öffentliche Verwaltung.

Kuttler, A. 1973. Gedanken über das Bodenrecht, dessen Gegenstand, Problematik und Entwicklung (Mskr. Verv.). Basel.

Laurent, Vincent. 2011. Perspectives et défis de la gestion durable du sous-sol en Suisse, Analyse des droits de propriété et d'usages relatifs à l'espace souterrain et aux éléments physiques du sous-sol. Cahier de l'IDHEAP 266. Chavannes-près-Renens: Hochschulinstitut für öffentliche Verwaltung.

Leimbacher, Jörg. 1995. Planungen und materielle Enteignung. Schriftenfolge der Schweizerischen Vereinigung für Landesplanung (VLP), Nr. 63. Bern.

Linder, Wolf. 1973. Der Fall Massenverkehr. Verwaltungsplanung und städtische Lebensbedingungen. Frankfurt: Athenäum.

Logan, John R., und Harvey L. Molotch. 1987. Urban Fortunes: the Political Economy of Place. Berkeley/Los Angeles: University of California Press. 
Mauch, Corine. 2001, Stadtentwicklung zwischen Plan und Markt. Cahier de l'IDHEAP 193/2001. Chavannes-près-Renens: Hochschulinstitut für öffentliche Verwaltung.

Molotch, Harvey L. 1999. Growth Machine Links: Up, down, and across. In The Urban Growth Machine: Critical Perspectives, Two Decades Later, Hrsg. Andrew E. G. Jonas und David Wilson, 247-265. Albany: State University of New York Press.

Moor, Pierre. 2002. L'expropriation matérielle. Droit administrative 2:741-755.

Nahrath, Stéphane. 2001. Les régimes institutionnels de la ressource sol entre 1870 et 2000. In Institutionelle Regime für natürliche Ressourcen: Boden, Wasser und Wald im Vergleich / Régimes institutionnels de ressources naturelles: analyse comparée du sol, de l'eau et de la forêt, Hrsg. Peter Knoepfel, Ingrid Kissling-Naef und Frédéric Varone, 49-99. Basel u. a.: Helbing \& Lichtenhahn.

Nahrath, Stéphane. 2003. La mise en place du régime institutionnel de l'aménagement du territoire en Suisse entre 1960 et 1990. Dissertation. Lausanne: IDHEAP / Universität Lausanne.

Nahrath, Stéphane. 2005. Le rôle de la propriété foncière dans la genèse et la mise en oeuvre de la politique d'aménagement du territoire: quels enseignements pour la durabilité des aménagements urbains? In Les enjeux $d u$ développement urbain durable. Forme urbaine, gestion des ressources et gouvernance, Hrsg. Antonio Da Cunha, Peter Knoepfel, Jean-Philippe Leresche und Stéphane Nahrath, 299-328. Lausanne: Presses Polytechniques et Universitaires Romandes.

Nahrath, Stéphane. 2008. Politique d'aménagement du territoire en Suisse: l'apport de la notion de régime institutionnel de ressources naturelles. In Territoires d'action. Aménagement, urbanisme, espace, Hrsg. Patrice Melé und Corinne Larrue, 63-94. Paris: L'Harmattan.

Nahrath, Stéphane, Peter Knoepfel, Patrick Csikos und Jean-David Gerber. 2009. Les stratégies politiques et foncières des grands propriétaires fonciers au niveau national. Etude comparée. Cahier de l'IDHEAP 246/2009. Chavannes-près-Renens: Hochschulinstitut für öffentliche Verwaltung.

Nicol, Lee Ann. 2009. Institutional Regimes for Sustainable Collective Housing. Dissertation. Lausanne: IDHEAP / Universität Lausanne.

Pfisterer, Thomas. 1975. Der Bundesbeschluss über dringliche Maßnahmen auf dem Gebiete der Raumplanung - eine Bilanz. Schweizerisches Zentralblatt für Staats- und Gemeindeverwaltung, ZBl 76:17-23.

Pollitt, Christopher, und Geert Bouckaert. 2004. Public Management Reform. A Comparative Analysis. Oxford: Oxford University Press.

Ratz, Michael. 1973. Bodenpolitik in der BRD. Kritik und demokratische Alternativen. Demokratie und Recht 16-48.

Rodewald, Raimund, und Peter Knoepfel. 2005. Institutionelle Regime für nachhaltige Landschaftsentwicklung, Série Ecologie \& Société, vol. 21. Zürich: Rüegger.

Ronge, Volker, und Günther Schmieg. 1972. Restriktionen politischer Planung, Bremen: Universität Bremen.

Rohr, Rudolf. 1966. Der Boden - ein Problem und seine Lösung. Genf: Redressement national.

Rohr, Rudolf. 1988. Tatsachen und Meinungen zur Bodenfrage. Freiheitliche Bodenordnung / Redressement National. Aarau: Verlag Sauerländer. 
Rotach, Martin. 1974. Rapport sur l'exécution de l'arrêté fédéral instituant des mesures urgentes en matière d'aménagement du territoire. Raumplanung Schweiz / Aménagement du territoire, April 1974. Bern: Département fédéral de justice et police.

Savary, Jérôme. 2008. Politiques publiques et mobilité urbaine / Analyse de processus conflictuels dans quatre villes suisses. Série Politikanalyse / Analyse des politiques publiques. Zürich: Rüegger.

Schwab, H.-P. 1996. Politique foncière communale: Ville de Bienne 1947-1996. Bienne: Administration des immeubles de la Ville de Bienne.

Schweizer, Rémi. 2010. Les bisses et leurs modes d'organisation au XXIe siècle, un modèle de gesetion durable ? Etude de cas à Savièse. Cahier de l'IDHEAP 257/2010. Chavannes-Lausanne: Hochschulinstitut für öffentliche Verwaltung.

Schweizerischer Bundesrat. 1967. Botschaft des Bundesrates über die Ergänzung der Bundesverfassung durch die Art. 22 ter und 22 quater (Verfassungsrechtliche Ordnung des Bodenrechts) vom 15.8.1967 (BBl. 1967 II 133 ff.).

Schweizerische Bundesverfassung, vom 18.4.1999, SR 101.

SNB (Schweizerische Nationalbank). 2010. Die Banken in der Schweiz. Zürich/Bern: SNB. SNB (Schweizerische Nationalbank). 2011. Bankenstatistisches Monatsheft April 2011. Zürich/Bern: SNB.

Soto, Hernando de. 2000. The Mystery of Capital: Why Capitalism Triumphs in the West and Fails Everywhere Else. New York: Basic Books.

Steiger Otto. 2006. Property Economics versus New Institutional Economics: Alternative Foundations of How to Trigger Economic Development. Journal of Economic Issues 40:183-208.

Stone, Clarence N. 1989. Regime Politics: Governing Atlanta, 1946-1988. Kansas City: University Press of Kansas.

Stone, Clarence N. 1993. Urban Regimes and the Capacity to Govern: A Political Economy Approach. Journal of Urban Affairs 15:1-28.

Stremlow, M., W. Zeh, Pia Klay und U. Roth. 1998. Landschaft zwischen gestern und morgen Grundlagen zum Landschaftskonzept Schweiz. Bundesamt für Umwelt, Wald und Landschaft / Bundesamt für Raumplanung.

Theurillat, Thierry, José Corpataux und Olivier Crevoisier. 2007. L'impact des caisses de pension sur les circuits de financement et de contrôle de l'économie suisse (1985-2003). Neuchâtel: Université de Neuchâtel, groupe de recherche en économie territoriale (GRET).

Tomerius, S., und C. Frick. 2009. Rechtliche Instrumente des Flächensparens unter besonderer Berücksichtigung der Paragraphen 1a Abs. 2 S.1 und 13a Abs. 1 S.1 BauGB.

Uhlmann, Björn, und Dietmar Braun. 2011. Die schweizerische Krankenversicherungspolitik zwischen Veränderung und Stillstand, Reihe Politikanalyse / analyse des politiques publiques. Chur: Rüegger.

van Griethuysen, Pascal. 2010. La RSE: nouvelle régulation du capitalisme ou interprétation capitaliste de la régulation? In Responsabilité de l'entreprise transnationale et globalisation de l'économie, Hrsg. Isabelle Daugareilh, 91-139. Brüssel: Bruyland.

UVEK. 2008. Bericht zur Totalrevision des Bundesgesetzes zur Raumplanung.

VLP-ASPAN. 2008a. Die Mehrwertabgabe nach Art. 5 RPG. Raum \& Umwelt 3/2008.

VLP-ASPAN. 2008b. Der schwierige Umgang mit Bauzonen. Inforaum(3). 
VLP-ASPAN. 2010. Fragen der Entschädigung im Falle von Rückzonungen im Zusammenhang mit der Teilrevision des RPG im Bereich der Siedlungsplanung. Raum \& Umwelt $6 / 2010$.

Vogl, Joseph. 2010/11. Das Gespenst des Kapitals. Zürich: diaphanes

Wagener, Frido. 1972. Öffentliche Planung und zukünftige politische Entscheidung. In Demokratie und Verwaltung, 25 Jahre Hochschule für Verwaltungswissenschaften Speyer. Schriftenreihe der Hochschule Speyer, Band 50, 571-588. Berlin: Duncker \& Humblot.

Walter, Françoise. 1985. Fédéralisme et propriété privée 1930-1950. Les attitudes face à l'aménagement du territoire en temps de crise et de pleins pouvoirs. In Dokumente und Informationen zur Schweizerischen Orts-, Regional- und Landesplanung (DISP), Nr. 82, Oktober 1985, 21-27. Zürich: ETH Zürich, Institut für Orts-, Regional- und Landesplanung.

Wenger, Anita. 2002. Die Baulandkontingentierung in der Raumplanungsgesetzgebung am Beispiel von Rückzonungen auf der Hueb (Berner Seeland). In Begrenzen um mehr zu erreichen. Kontingente als Instrumente der Umwelt- und Raumordnungspolitik, Hrsg. Peter Knoepfel, 255-320. Bern: EPA.

Winter, Gerd. 2007. Natur ist Fundament, nicht Säule. Zwanzig Jahre nachhaltige Entwicklung als rechtspolitisches Konzept. Gaia 16:225-260

Zuppinger, Urs. 1990. Vers un nouveau type de règlement du plan d'affectation communal : effets des lois et règlements d'urbanisme existant sur la consommation du sol et la structure d'affectation des zones bâties, proposition et évaluation d'une approche alternative, Bericht 46 „Boden“. Liebefeld-Bern: Programme national de recherche.

Zuppinger, Urs. 2012. Luttes-ô-Flon, une reconversion urbaine lausannoise mouvementée de 1984 à 2012. Lausanne: édition d'en bas.

\section{Autorenangaben:}

Prof. Dr. Peter Knoepfel,

Institut de hautes études en administration publique (IDHEAP), Université de Lausanne, 1015 Lausanne, Suisse, peter.knoepfel@idheap.unil.ch

[Korrespondenzautor]

Patrick Csikos, MPA, Institut Universitaire Kurt Bösch (IUKB), Case postale 4176, 1950 Sion 4, Suisse, patrick.csikos@iukb.ch

Assistenzprofessor Dr. Jean-David Gerber, Geographisches Institut der Universität Bern (GIUB), Hallerstrasse 12, 3012 Bern, Schweiz, jean-david.gerber@unil.ch

Prof. Dr. Stéphane Nahrath, Institut Universitaire Kurt Bösch (IUKB), Case postale 4176, 1950 Sion 4, Suisse, stephane.nahrath@iukb.ch 